$\underline{\text { Preprint typeset in JHEP style - PAPER VERSION }}$

CERN-PH-TH/2005-022

FTUV-05/0208

IFIC/05-14

\title{
The Scherk-Schwarz mechanism as a flux compactification with internal torsion
}

\author{
L. Andrianopoli \\ Centro E. Fermi, Compendio Viminale, I-00184 Rome, Italy \\ E-mail: Laura.Andrianopoli@cern.ch
}

\author{
M. A. Lledó \\ Departament de Física Teòrica, Universitat de València and IFIC, C/Dr. Moliner, 50, \\ E-46100 Burjassot (València), Spain. \\ E-mail: Maria.Lledo@ific.uv.es
}

\section{Trigiante \\ Dipartimento di Fisica, Politecnico di Torino, C.so Duca degli Abruzzi, 24 I-10129 Torino, Italy \\ E-mail: Mario.Trigiante@to.infn.it}

\begin{abstract}
The aim of this paper is to make progress in the understanding of the ScherkSchwarz dimensional reduction in terms of a compactification in the presence of background fluxes and torsion. From the eleven dimensional supergravity point of view, we find that a general $\mathrm{E}_{6(6)}$ S-S phase may be obtained by turning on an appropriate background torsion, together with suitable fluxes, some of which can be directly identified with certain components of the four-form field-strength. Furthermore, we introduce a novel (four dimensional) approach to the study of dualities between flux/torsion compactifications of Type II/M-theory. This approach defines the action that duality should have on the background quantities, in order for the $\mathrm{E}_{7(7)}$ invariance of the field equations and Bianchi identities to be restored also in the presence of fluxes/torsion. This analysis further implies the interpretation of the torsion flux as the T-dual of the NS three-form flux.
\end{abstract}




\section{Contents}

1. Introduction 1

2. Coupling of torsion to gauge fields: an example 4

3. Dimensional reduction in the presence of a torsion background 6

3.1 Dimensional reduction with a torsionless connection 6

$\begin{array}{lll}3.2 & \text { The role of torsion } & 7\end{array}$

3.3 The $D$ dimensional Lagrangian in the presence of torsion. 12

4. Other couplings in maximal S-S supergravity from internal torsion. 12

5. Completion of the S-S phase $\quad 14$

$\begin{array}{ll}\text { 6. A four dimensional analysis } & 17\end{array}$

7. Conclusions 23

A. Some useful relations and definitions about the torsion 24

A.1 Decomposition of $\omega_{B}^{A}$ in terms of $D$-dimensional fields 25

A.2 D-dimensional Kaluza-Klein vectors 26

\section{Introduction}

This note is intended to shed light on the interpretation of the Scherk-Schwarz mechanism for generalized dimensional reduction [1] within the framework of string compactifications in the presence of background fluxes and a non-trivial background torsion in the internal manifold.

Recently there has been considerable interest in the construction of phenomenologically viable string models. The role of a warped metric in generating a large hierarchy of scales, fixed in terms of charges determined by RR and NS background fluxes, has been extensively studied in the literature [3,4]. A particular attention has been devoted to microscopic settings leading to a low energy action with a zero vacuum energy. Models of this kind are generalized no-scale models [5,6] which are typically related to gaugings of suitable non-semisimple global symmetry groups of the Lagrangian (flat gaugings) $[7,8]$. They have been obtained from flux compactifications, mostly in Type IIB theory by switching on appropriate RR and NS three-form fluxes in the internal directions $[10,11,12]$ (fewer examples were obtained on the Type IIA front $[13,14]$ ), as well as from generalized dimensional reduction à la Scherk-Schwarz (S-S) $[1,2,4,8,9,15,16]$ of eleven dimensional 
supergravity (or any truncation thereof). This is a generalized type of dimensional reduction on tori, which induces non abelian couplings and a positive definite scalar potential. Generally, it yields to spontaneous supersymmetry breaking. The simplest example is a model describing a single complex scalar field $\phi(x, y)$ on $\mathbb{R}^{3,1} \times S^{1}$, with Lagrangian

$$
\mathcal{L}=\frac{1}{2} \partial_{\hat{\mu}} \phi \partial^{\hat{\mu}} \phi^{*} ; \quad \hat{\mu}=(\mu, 4) ; \quad \mu=0, \ldots, 3 ; x^{4}=y .
$$

$\mathcal{L}$ is invariant under a global $U(1): \phi \rightarrow \mathrm{e}^{\mathrm{i} \alpha} \phi$, and this allows a generalized compactification ansatz:

$$
\phi(x, y)=\mathrm{e}^{\mathrm{i} m y} \sum_{n=-\infty}^{+\infty} \phi_{n}(x) \mathrm{e}^{\mathrm{i} n y / 2 \pi R} .
$$

We note that the multivaluedness of $\phi(x, y)$ on $S^{1}$ does not pose problems in the definition of the lower dimensional theory, since, being the phase $\mathrm{e}^{\mathrm{i} 2 \pi m R}$ a global symmetry of the original theory, it finally cancels in the Lagrangian. The only effect of the phase is to shift the four dimensional mass spectrum, so that the zero-mode has mass $m$. This mechanism may be applied to the case of $D=5, N=8$ supergravity [2], whose scalars span the manifold

$$
\frac{\mathrm{E}_{6(6)}}{\mathrm{USp}(8)} \subset \frac{\mathrm{E}_{7(7)}}{\mathrm{SU}(8)}
$$

The S-S dimensional reduction can be performed by using, as global symmetry, any subgroup of the 78-dimensional global symmetry group $\mathrm{E}_{6(6)}$. We shall call $S$ - $S$ generator a generator of the global symmetry transformation entering the S-S ansatz. It is known that only if the S-S generators are compact, the resulting no-scale model admits a (Minkowski) vacuum. For any other choice, it can be shown that the corresponding no-scale scalar potential is of run-away type.

As the $\mathrm{E}_{6(6)}$ global symmetry of the $N=8, D=5$ supergravity is manifest when the theory is obtained by dimensional reduction on a torus of eleven dimensional supergravity, this pattern should naturally be embedded in the framework of M-theory, or of Type IIA supergravity. There is an extensive literature on the subject, which includes Refs. $[14,17,18]$.

However a precise interpretation of the S-S phases in terms of microscopic (stringy) objects has not been completely understood, since it seems to include non perturbative stringy degrees of freedom.

On the other hand, as mentioned above, the pattern of flux compactification has proven to be successful in providing viable models with flat gaugings mostly for Type IIB theory. Some of these models have then been reinterpreted in terms of Type IIA theory by exploiting T-duality, although the proper generalization of this correspondence to vacua in which background fluxes are present is not yet thoroughly understood. For instance it was soon apparent that the T-dual of the NS three-form flux in Type II theory could not, in general, be found among the fluxes associated to the ten-dimensional p-forms of the string spectrum. There is strong evidence that a non-vanishing torsion in the background geometry of the internal manifold should play an important role in filling this gap and thus completing the duality picture connecting different superstring flux-vacua (the problem of 
generalizing mirror symmetry in the presence of fluxes was addressed in $[20,9,21]$ while for a general analysis of compactifications on manifolds with torsion see [19, 20, 22]).

The main purpose of the present paper is to show how theories originating from a S-S reduction from one dimension higher, for a suitable choice of the S-S generator, can be alternatively interpreted as the result of a toroidal dimensional reduction in the presence of an internal torsion. In the case of maximal S-S supergravity in four dimensions we shall also speculate, by using group theory techniques, on the possible M-theory flux interpretation of (part of) the remaining parameters of the global symmetry group.

The paper is organized as follows:

In Section 2 we shall anticipate our idea with a toy model.

In Section 3 we shall consider a $D+n$ dimensional pure gravity theory compactified on a torus $T^{n}$ and dimensionally reduced to $D$ space-time dimensions. We are going to show that, by switching on an appropriate constant background torsion in the internal $n$-torus $T^{n}$ (a torsion flux), we obtain the same $D$-dimensional theory as the one originated by a S-S reduction from $D+1$ dimensions. The S-S generators are chosen within the global symmetry algebra $\mathfrak{s l}(n-1, \mathbb{R})$.

In Section 4 we shall extend this analysis to the $D=4$ maximal supergravity theory obtained from eleven dimensions by dimensional reduction. We show that the couplings that would appear, if performing a S-S reduction from $D=5$ with a $\mathrm{SL}(6, \mathbb{R})$ phase, can be interpreted in terms of a standard dimensional reduction where the internal manifold is given a torsion.

In Section 5, we will turn to analyze the microscopic interpretation of the $N=8, D=4$ supergravity obtained through a S-S reduction from $D=5$. Since the global symmetry group in 5 dimensions has 78 parameters, a general S-S phase can depend on all of them. We shall face the problem of interpreting these parameters in terms of eleven dimensional background fluxes and torsion. Besides the 36 parameters originating from the internal torsion, and which correspond to the choice of the S-S generator within the $\mathfrak{g l}(6, \mathbb{R})$ global symmetry algebra in $D=5$, we find, for 21 additional parameters, a direct and simple interpretation in terms of the eleven dimensional 4-form field strength, corresponding to the choice of the S-S generator in the remaining (nilpotent) part of the Borel subalgebra of $\mathfrak{e}_{6(6)}$.

In Section 6, we introduce the embedding tensor approach to gauged maximal supergravities, which is based on the description of these models in terms of the so called T-tensor, first introduced in the literature in Ref. [23]. In particular we use a fourdimensional mathematical framework in which background fluxes and/or torsion are described as components of the embedding matrix defining the corresponding gauged supergravity. This identification, together with the characterization made in Ref. [24] of the embedding matrix as an $\mathrm{E}_{7(7)}$ covariant tensor (transforming in the 912), will allow to describe, in maximal four dimensional supergravities originating from Type II or M-theory, internal background fluxes and torsion as elements of a larger $\mathrm{E}_{7(7)}$ representation. As a consequence of this mathematical characterization, in gauged lower dimensional maximal supergravities originating from flux/torsion compactification, the duality symmetry of the ungauged field equations and Bianchi identities can be restored if, besides the fields, 
the background quantities are transformed under duality as well. A similar analysis was made in Ref. [4] in the context of Heterotic theory compactifications. According to our assumptions, we shall show that internal flux and/or torsion components can indeed be consistently identified with elements of the larger $E_{7(7)}$ representation in which the embedding tensor transforms. A rule for associating flux and torsion components with $\mathrm{E}_{7(7)}$ weights is given. This analysis provides a nice mathematical laboratory where to study the effects of dualities on flux-vacua (including the presence of internal torsion as a flux of the metric moduli). As a byproduct of this, we show that the torsion flux provides precisely the T-dual of the NS three-form flux.

We refer the reader to the Appendix for a formal definition of torsion and most of the technical details.

\section{Coupling of torsion to gauge fields: an example}

The crucial point in our investigation is the coupling of fields with non vanishing spin to gravity, in the presence of a torsion background.

Let us consider, as an example, the case of a vector field $A_{M}$. The principle of general covariance demands that the field strength is computed in terms of covariant derivatives of the torsionfull affine connection $\tilde{\nabla}$ (see the Appendix for definitions and conventions):

$$
F_{M N} \equiv \tilde{\nabla}_{[M} A_{N]}=\partial_{[M} A_{N]}+T_{M N}^{P} A_{P}
$$

When $T \neq 0 F$ is not invariant under the usual gauge transformations

$$
\delta A_{M}(X)=\partial_{M} \Lambda(X)
$$

However, it is possible [25] to give a generalized definition of gauge invariance which makes the torsion compatible with the presence of gauge fields. The generalized gauge transformation of the field $A_{M}$ has the form

$$
\delta A_{M}(X)=C_{M}^{N}(X) \partial_{N} \Lambda(X)
$$

where the point-dependent matrix $C_{M}^{N}(X)$ has to be constrained by the request of gauge invariance of the field strength

$$
\delta F_{M N}=0 .
$$

This procedure will be reviewed in section 3.2. The general result, found in Ref. [25], for a gauge field in $d$ dimensions coupled to torsion in $d$ dimensions is ${ }^{1}$

$$
C_{M}^{N}=\delta_{M}^{N} e^{\phi}, \quad T_{M N}^{P}=\delta_{[M}^{P} \partial_{N]} \phi
$$

Note that the torsion tensor in (2.5) appears to be of a restricted form. There is only one torsion degree of freedom allowed, the scalar field $\phi$.

\footnotetext{
${ }^{1}$ We use throughout the paper the notation $q_{(A B)}=\frac{1}{2}\left(q_{A B}+q_{B A}\right)$ to indicate symmetrization of the indices, and correspondingly $q_{[A B]}=\frac{1}{2}\left(q_{A B}-q_{B A}\right)$ to indicate antisymmetrization.
} 
As we will see, by combining this idea with the dimensional reduction procedure, we can relax the stringent conditions (2.5). In fact, in the spirit of the dimensional reduction from $D+n$ to $D$ dimensions it is enough to require gauge invariance of the $D$-dimensional theory.

The main goal of this paper will be to show that this procedure reproduces the S-S mechanism, thus providing a geometrical interpretation for it. This will be done in section 3.2 .

For the time being, let us illustrate our idea with a toy model, where the metric, together with the torsion, are non dynamical, background fields. The torsion is assumed to satisfy (2.5), so it is given in terms of a scalar field $\phi$. We consider the five dimensional space-time $\mathbb{R}^{1,3} \times \mathrm{S}^{1}$, with coordinates $x^{M}, M=0, \ldots 4$, which we split as $X^{M}=\left(x^{\mu}, y\right)$ with $\mu=0, \ldots, 3$ and the fifth dimension compactified on a circle. For the field $\phi$ we take

$$
\phi=\phi(y)=m y, \quad m=\text { constant } .
$$

$\phi$ is multivalued in the circle, but the torsion is not. With these premises we obtain

$$
F_{M N}=\partial_{[M} A_{N]}+\partial_{[M} \phi A_{N]}=\partial_{[M} A_{N]}-m \delta_{[M}^{4} A_{N]},
$$

with gauge transformations

$$
\delta A_{M}=e^{m y} \partial_{M} \Lambda .
$$

We would like to make a dimensional reduction and assume $A_{M}(X)=A_{M}(x)$. But given the gauge transformations allowed for the field $A_{M}(2.8)$, this choice cannot be preserved by a gauge transformation. The effect of the torsion on the circle is then to give effectively to the gauge vector a dependence on $y$. This dependence can be chosen of the form,

$$
A_{\mu}(x, y)=e^{m y} A_{\mu}^{0}(x),
$$

which is preserved by gauge transformations.

If we want to include all the Kaluza-Klein modes, we have that the theory compactified on the circle contains a tower of vectors

$$
A_{\mu}(x, y)=e^{m y} \sum_{k} e^{i k y} A_{\mu}^{k}(x),
$$

and scalars

$$
A_{4}(x, y)=e^{m y} \sum_{k} e^{i k y} A_{4}^{k}(x) .
$$

We immediately see that the effect of the internal torsion has been to generate an exponential factor à la Scherk-Schwarz. Indeed, the field strength for the zero-mode splits as

$$
\begin{aligned}
& F_{\mu \nu}=\partial_{[\mu} A_{\nu]}, \\
& F_{\mu 4}=\frac{1}{2}\left(\partial_{\mu} A_{4}-m A_{\mu}\right)=\frac{1}{2} e^{m y} D_{\mu} A_{4}^{0},
\end{aligned}
$$

where $D_{\mu}$ denotes a covariant derivative with respect to the four dimensional gauge connection $A_{\mu}^{0}$. Then, the four dimensional vector $A_{\mu}^{0}$ gauges the translational isometry of the action along the direction of the the axion $A_{4}^{0}$. 


\section{Dimensional reduction in the presence of a torsion background}

\subsection{Dimensional reduction with a torsionless connection}

Let us consider a gravity theory in $D+n$ dimensions. We denote by $X^{M}, M=0,1, \cdots, D+$ $n-1$, the coordinates in the space-time manifold. We will split the indices as $X^{M}=$ $\left(x^{\mu}, y^{\underline{m}}\right)$ where the coordinates $x^{\mu}, \mu=0,1, \cdots D-1$, parametrize non compact directions, while $y \underline{m}, \underline{m}=1, \cdots n$, parametrize the $n$ directions of some compact manifold $\mathcal{M}_{n}$. Here and in the following, we will take $\mathcal{M}_{n}=T^{n}$. The generalization to more general internal manifolds will be discussed elsewhere. We will further split the index $\underline{m}=(D, m)$ with $m=1, \ldots n-1$.

The metric in the $D+n$-dimensional manifold can be conveniently written in terms of the unconstrained fields

$$
G_{M N}=\left(\begin{array}{cc}
e^{\frac{2 \sigma}{2-D}} g_{\mu \nu}+G_{\underline{m n}} B \frac{m}{\mu} B_{\bar{\nu}}^{\frac{n}{\nu}} & G_{\underline{m n}} B \frac{n}{\mu} \\
G_{\underline{m n}} B_{\bar{\nu}}^{\frac{m}{\nu}} & G_{\underline{m n}}
\end{array}\right)=\mathcal{V}_{M}^{A} \eta_{A B}\left(\mathcal{V}^{T}\right)^{B} .
$$

$\eta_{A B}$ is the flat metric, defined with mostly + signs. $\mathcal{V}^{A}=\mathcal{V}_{M}^{A} d X^{M}$ is an orthonormal frame (vielbein). The flat (tangent space) indices $A=1, \cdots, D+n$ split also as

$$
A=(a, \underline{i})=(a, \hat{D}, i), \quad a=0, \ldots D-1, \quad i=1, \ldots n-1 .
$$

After using partially the $\mathrm{SO}(1, D+n-1)$ gauge freedom, the vielbein can be written as

$$
\mathcal{V}_{M}^{A}=\left(\begin{array}{ccc}
e^{\frac{\sigma}{2-D}} V_{\mu}{ }^{a} & V_{D} \hat{D}^{\hat{D}} B_{\mu}^{D} & V_{\underline{m}^{i}}^{i} B^{\frac{m}{\mu}} \\
0 & V_{D} \hat{D} & V_{D}^{i} \\
0 & 0 & V_{m}^{i}
\end{array}\right)
$$

and its inverse is

$$
\mathcal{V}_{A}^{M}=\left(\begin{array}{ccc}
e^{-\frac{\sigma}{2-D}} V_{a}^{\mu}-e^{-\frac{\sigma}{2-D}} V_{a}^{\mu} B_{\mu}^{D} & -e^{-\frac{\sigma}{2-D}} V_{a}^{\mu} B_{\mu}^{m} \\
0 & V_{\hat{D}}{ }^{m} & -V_{\hat{D}}{ }^{D} V_{D}{ }^{i} V_{i}^{m} \\
0 & 0 & V_{i}^{m}
\end{array}\right) .
$$

The vielbein of the compact manifold $\mathcal{M}_{n}$ and its inverse are $\operatorname{GL}(n, \mathbb{R})$ matrices

$$
V_{\underline{\underline{n}}}^{\underline{i}} \equiv\left(\begin{array}{cc}
V_{D}^{\hat{D}} & V_{D}^{i} \\
0 & V_{n}^{i}
\end{array}\right) ; \quad V_{\underline{i}}^{\underline{m}} \equiv\left(\begin{array}{cc}
V_{\hat{D}}^{D} & V_{\hat{D}}^{m} \\
0 & V_{i}^{n}
\end{array}\right) \text {. }
$$

They satisfy

$$
V_{\underline{\underline{m}}}^{\underline{i}} \eta_{\underline{i j}} V_{\underline{\underline{n}}}^{\underline{j}}=G_{\underline{m n}}=e^{\frac{2}{n} \sigma} g_{\underline{m n}}, \quad \text { with } \quad \operatorname{det}\left(g_{\underline{m n}}\right)=1 .
$$

The $\mathrm{SO}(1,1)$ field $e^{\sigma}$ corresponds to the volume of the internal manifold, and it has been treated separately such as to have a canonical Einstein term in the Lagrangian in $D$ dimensions ${ }^{2}$.

\footnotetext{
${ }^{2}$ Indeed, with this definition one has $\sqrt{\operatorname{det}\left(G_{D+n}\right)}=e^{\left(1+\frac{D}{2-D}\right) \sigma} \sqrt{\operatorname{det}\left(g_{D}\right)}$ and $\mathcal{R}_{D}=R_{b \mu \nu}^{a} \mathcal{V}_{a}{ }^{\mu} \mathcal{V}_{\rho}^{b} G^{\rho \nu}+\cdots \sim e^{\frac{-2 \sigma}{2-D}} \quad$ so that $\quad \sqrt{\operatorname{det}\left(G_{D+n}\right)} \mathcal{R}_{D+n}=\sqrt{\operatorname{det}\left(g_{D}\right)} \mathcal{R}_{D}$
} 
We make the Kaluza-Klein ansatz, and we truncate the Kaluza-Klein spectrum to the 0 -modes, so that

$$
\mathcal{V}_{M}^{A}=\mathcal{V}_{M}^{A}(x)
$$

After substituting (3.6), the components of the torsionless spin connection (A.7) $\omega^{A B}$ decompose as (we use flat indices)

$$
\begin{aligned}
\omega_{a b, c} & =e^{-\frac{\sigma}{2-D}}\left(\frac{2}{2-D} \partial_{[a} \sigma \eta_{b] c}+\bar{\omega}_{a b, c}\right), \\
\omega_{a b, \underline{i}} & =e^{-\frac{2 \sigma}{2-D}} \partial_{[a} B \frac{m}{b]} V_{\underline{m i}}, \\
\omega_{a \underline{i}, b} & =\omega_{a b, \underline{i}}, \\
\omega_{a \underline{i}, \underline{j}} & =e^{-\frac{\sigma}{2-D}}\left(P_{\underline{i} \underline{j}, a}+\frac{1}{n} \eta_{\underline{i} \underline{j}} \partial_{a} \sigma\right)=\omega_{a \underline{j}, \underline{i}}, \\
\omega_{\hat{D} i, a} & =-\omega_{a \hat{D}, i}, \\
\omega_{\hat{D} i, \underline{j}} & =0 \\
\omega_{i j, a} & =-e^{-\frac{\sigma}{2-D}} V_{[i}^{m} \partial_{a} V_{m \mid j]} \equiv-e^{-\frac{\sigma}{2-D}} Q_{i j, a}, \\
\omega_{i j, \underline{k}} & =0
\end{aligned}
$$

where we have used the definitions

$$
\partial_{a} \equiv V_{a}^{\mu} \partial_{\mu}, \quad e^{a} \equiv V_{\mu}^{a} d x^{\mu}
$$

for dual basis in the tangent and cotangent spaces, and

$$
\left(V^{-1} d V\right)_{\underline{i j}}=\left(V^{-1} d V\right)_{\underline{i} \underline{j}, a} e^{a} \equiv Q_{\underline{i j}}+P_{\underline{i} \underline{j}}+\frac{1}{n} \eta_{\underline{i} \underline{j}} d \sigma
$$

with

$$
\begin{aligned}
P_{\underline{i} \underline{j}} & =\left(V^{-1} d V\right)_{(\underline{i j})}-\frac{1}{n} \eta_{\underline{i} \underline{j}} \operatorname{tr}\left(V^{-1} d V\right) \quad \text { so } \quad P_{\underline{i} \underline{j}} \eta^{\underline{i} \underline{j}}=0, \\
Q_{\underline{i} \underline{j}} & =\left(V^{-1} d V\right)_{[\underline{i} \underline{j}]} .
\end{aligned}
$$

Finally, $\bar{\omega}_{a b, c}=\bar{\omega}_{a b, \mu} V_{c}{ }^{\mu}$ is the torsionless spin connection of the $D$-dimensional space-time.

\subsection{The role of torsion}

We want to include now the effect of a torsion background.

Let $\Omega_{B}^{A}=\omega_{B}^{A}+\Delta \Omega_{B}^{A}$ be an antisymmetric spin-connection, with $\omega_{B}^{A}$ its torsionless part. The torsion tensor is (see the Appendix)

$$
T^{A}=d \mathcal{V}^{A}+\Omega_{B}^{A} \wedge \mathcal{V}^{B}=\Delta \Omega_{B}^{A} \wedge \mathcal{V}^{B}
$$

We make the following ansatz for the dependence of the torsion on space-time:

$$
T_{M N}^{P}=T_{M N}^{A} \mathcal{V}_{A}^{P}=T_{M N}^{P}\left(X^{D}\right),
$$

that is, we ask that the torsion tensor depends only on the coordinate $X^{D}=y^{1}$. 
As we discussed in section 2, in the presence of torsion the field strengths of fields with non-zero spin get modified as in (2.1) (due to the principle of general covariance) because of the antisymmetric part in the affine connection.

Let us consider, for instance, the case of a gauge vector $A$. When $T \neq 0$, its fieldstrength $F$ is not gauge invariant. However, with the generalized prescription for gauge transformations (2.3), that we introduced in section 2 , it is possible to achieve the gauge invariance of the field strength

$$
\delta F_{M N}=0,
$$

thus making the torsion compatible with the presence of gauge fields [25].

On the other hand, since we would like to make the Kaluza-Klein ansatz for all the spectrum, for our purpose it is sufficient to assume, for the gauge parameter

$$
\Lambda=\Lambda(x)
$$

while allowing for $C_{M}^{N}$, which is associated to the presence of the torsion, a dependence on the coordinate $X^{D}=y^{1}$

$$
C_{M}^{N}=C_{M}^{N}\left(X^{D}\right)
$$

As proven in [25], the request of gauge invariance (in the generalized sense) of the fieldstrength is achieved for the generalized gauge transformation (2.3), under the following condition

$$
\delta F_{M N}=C_{[N}^{P} \partial_{M]} \partial_{P} \Lambda+\left(\partial_{[M} C_{N]}^{P}+T_{M N}^{R} C_{R}^{P}\right) \partial_{P} \Lambda=0 .
$$

With the given ansatz (3.14), this condition corresponds to the equations

$$
\begin{aligned}
& C_{N}^{P} \delta_{M}^{(\mu} \delta_{P}^{\nu)}-C_{M}^{P} \delta_{N}^{(\mu} \delta_{P}^{\nu)}=0, \\
& \left(\partial_{[M} C_{N]}^{P}+T_{M N}^{R} C_{R}^{P}\right) \delta_{P}^{\mu}=0 .
\end{aligned}
$$

They may be solved for tensors $C_{M}^{N}$ and $T_{M N}^{P}$ with the only non-zero entries

$$
\begin{aligned}
C_{M}^{N} & :\left(C_{\mu}^{\nu}, C_{\mu} \frac{n}{}, C_{\underline{\underline{m}}}\right), \\
T_{M N}^{P} & :\left(T_{\nu D}^{\mu}, T^{\underline{p}}{ }_{M N}\right),
\end{aligned}
$$

with the constraints:

$$
C_{\mu}{ }^{\nu}=\delta_{\mu}^{\nu} e^{\phi} ; \quad T_{\nu D}^{\mu}=\delta_{\nu}^{\mu} \frac{1}{2} \partial_{D} \phi
$$

We note that the other non-zero entries are not restricted.

In particular, we may consider a torsion tensor whose only non-zero components are

$$
T_{\nu D}^{\mu}=\frac{1}{2} \delta_{\nu}^{\mu} \partial_{D} \phi ; \quad T^{m}{ }_{n D}=-\frac{1}{2} \partial_{D} \Phi_{n}^{m},
$$

where $\phi=\phi\left(X^{D}\right), \Phi_{n}^{m}=\Phi_{n}^{m}\left(X^{D}\right)$. The tensor $\Phi$ is taken to be a matrix of $\mathfrak{s l}(n-1, \mathbb{R})$.

The first contribution to the torsion, $T^{\mu}{ }_{\nu D}$, is in fact a warping factor, which can be interpreted as an extra, $y^{1}$-dependent contribution to the dilaton $\sigma$. Indeed, it gives

$$
d \mathcal{V}^{a}+\omega_{b}^{a} \wedge \mathcal{V}^{b}-\frac{1}{2} d \phi \wedge \mathcal{V}^{a}=0
$$


that is

$$
d\left(e^{\frac{1}{2} \phi} \mathcal{V}^{a}\right)+\omega_{b}^{a} \wedge\left(e^{\frac{1}{2} \phi} \mathcal{V}^{b}\right)=0 .
$$

We discard such contribution (setting $\phi=0$ ) because one can see that it induces non abelian couplings for the vectors $B^{m}$ which are not compatible with the definition of covariant derivatives for the scalars. It would then introduce ghosts in the theory.

The ansatz that we make for the non zero components of the torsion is:

$$
T^{m}{ }_{n D}=-\frac{1}{2} \partial_{D} \Phi^{m}{ }_{n}
$$

We are going to show that the given ansatz for the torsion, with $\Phi_{n}^{m} \in \mathfrak{s l}(n-1)$, precisely reproduces the Scherk-Schwarz mechanism with a phase $M \in \mathfrak{s l}(n-1) \subset \mathfrak{e}_{n-1(n-1)}$.

Let us note that then eq. (2.3) becomes, for any choice of tensor $C_{\underline{\underline{m}}}$,

$$
\delta A_{\mu}=\partial_{\mu} \Lambda, \quad \delta A_{\underline{m}}=0,
$$

which is compatible (differently from the toy model case) with the Kaluza-Klein ansatz $A_{M}=A_{M}(x)$.

With our ansatz, any vector field strength $F_{M N}=\tilde{\nabla}_{[M} A_{N]}$ of the $D+n$-dimensional theory decomposes into the $D$-dimensional fields:

$$
\begin{aligned}
F_{\mu \nu} & =\partial_{[\mu} A_{\nu]}, \\
F_{\mu \underline{n}} & =\frac{1}{2} \partial_{\mu} A_{\underline{n}}, \\
F_{D n} & =\frac{1}{2} \partial_{D} \Phi_{n}^{m} A_{m} .
\end{aligned}
$$

Let us observe that, in order for these relations to be compatible with the Kaluza-Klein ansatz, we have to further restrict the torsion to be a constant:

$$
\partial_{D} \Phi_{n}^{m}=M_{n}^{m}=\text { constant; } \quad\left(M_{m}^{m}=0\right) .
$$

Then, in the model that we are considering the torsion $T_{M N}^{P}$ is a constant tensor.

In the rest of this section we will study the effects of the torsion (3.25) on the $D$ dimensional fields coming from the metric in $(D+n)$ dimensions.

In the presence of torsion, the Riemann tensor has extra contributions

$$
\tilde{R}_{B}^{A}=d \Omega_{B}^{A}+\Omega_{C}^{A} \wedge \Omega_{B}^{C}=R_{B}^{A}+\left(d_{\nabla} \Delta \Omega\right)_{B}^{A}+\Delta \Omega_{C}^{A} \wedge \Delta \Omega_{B}^{C},
$$

where the symbol $d_{\nabla}$ means covariant differentiation with respect to the torsionless part $\omega$ of the spin connection, given in (3.7).

As it is shown in the Appendix, one finds

$$
\Delta \Omega_{B \mid N}^{A}=K_{B \mid N}^{A},
$$

where

$$
K_{B N}^{A}=\mathcal{V}_{P}^{A} \mathcal{V}_{M}^{B} K^{P}{ }_{M N}=\mathcal{V}_{P}{ }^{A} \mathcal{V}_{B}{ }^{M}\left(T^{P}{ }_{M N}-T_{M}{ }^{P}{ }_{N}-T_{N}{ }^{P}{ }_{M}\right),
$$


that is

$$
\begin{aligned}
K_{A B, C} & =\left(\mathcal{V}_{P A} \mathcal{V}_{B}{ }^{M} \mathcal{V}_{C}{ }^{R}-\mathcal{V}_{A}{ }^{M} \mathcal{V}_{P B} \mathcal{V}_{C}{ }^{R}+\mathcal{V}_{A}{ }^{R} \mathcal{V}_{B}{ }^{M} \mathcal{V}_{P C}\right) T^{P}{ }_{M R} \\
& =M_{n}^{m}\left(\mathcal{V}_{m[A} \mathcal{V}_{B]}^{D} \mathcal{V}_{C}^{n}-\mathcal{V}_{[A}{ }^{D} \mathcal{V}_{B]}{ }^{m} \mathcal{V}_{n C}-\mathcal{V}_{m[A} \mathcal{V}_{B]}{ }^{n} \mathcal{V}_{C}{ }^{D}\right)
\end{aligned}
$$

where in the last expression we have used our ansatz (3.25) for the torsion. Let us use the following short-hand notation:

$$
B \frac{m}{a}=V_{a}^{\mu} B \frac{m}{\mu}
$$

We get:

$$
\begin{aligned}
K_{a b, c} & =0 \\
K_{a b, \hat{D}} & =0 \\
K_{a b, i} & =-e^{-\frac{2 \sigma}{2-D}} M_{n}^{m} B_{[a}^{D} B_{b]}^{n} V_{m i}, \\
K_{a \hat{D}, b} & =0 \\
K_{a \hat{D}, \hat{D}} & =0 \\
K_{a \hat{D}, i} & =-\frac{1}{2} e^{-\frac{\sigma}{2-D}} M_{n}^{m}\left(V_{\hat{D}}^{D} B_{a}^{n}-B_{a}^{D} V_{\hat{D}}^{n}\right) V_{m i}, \\
K_{a i, b} & =K_{a b, i} \\
K_{a i, \hat{D}} & =K_{a \hat{D}, i}, \\
K_{a i, j} & =e^{-\frac{\sigma}{2-D}} M^{m}{ }_{n} B_{a}^{D} V_{m(i} V_{j)}{ }^{n} \\
K_{\hat{D} i, a} & =-K_{a \hat{D}, i}, \\
K_{\hat{D} i, \hat{D}} & =0, \\
K_{\hat{D} i, j} & =-M_{n}^{m}{ }_{n} V_{\hat{D}}^{D} V_{m(i} V_{j)}^{n} \\
K_{i j, a} & =e^{-\frac{\sigma}{2-D}} M^{m}{ }_{n} V_{m[i} V_{j]}^{n} B_{a}^{D}, \\
K_{i j, \hat{D}} & =-M_{n}^{m}{ }_{m[i} V_{j]}{ }^{n} V_{\hat{D}}^{D} \\
K_{i j, k} & =0 .
\end{aligned}
$$

We introduce now the structure constants $f \frac{m}{\underline{n} p} \underline{\text { defined as }}$

$$
f_{D n}^{m}=-f_{n D}^{m}=-M_{n}^{m}, \quad \text { and the rest zero, }
$$

satisfying the Jacobi identities. We can then define the non abelian field-strengths

$$
F^{\underline{m}} \equiv d B^{\underline{m}}+\frac{1}{2} f \frac{\underline{m}}{\underline{n} \underline{p}} B^{\underline{n}} \wedge B^{\underline{p}},
$$

or more explicitly

$$
\begin{aligned}
F_{a b}^{D} & \equiv \partial_{[a} B_{b]}^{D}, \\
F_{a b}^{m} & \equiv \partial_{[a} B_{b]}^{m}+f_{D n}^{m} B_{[a}^{D} B_{b]}^{n} .
\end{aligned}
$$


Furthermore, let us define the gauge covariant derivative of a generic scalar $s_{\underline{m}}$ with a covariant internal index $\underline{m}$ as

$$
D_{a} s_{\underline{m}} \equiv \partial_{a} s_{\underline{m}}+f_{\underline{m} \underline{p}}^{n} B \frac{p}{a} s_{n}
$$

which then allows the definitions

$$
\begin{aligned}
& \hat{P}_{\underline{i j, a}}=V_{(\underline{i}} \underline{m} D_{a} V_{\underline{m} \mid \underline{j})}-\frac{1}{n} \eta_{\underline{i j}} \operatorname{tr}\left(V_{(\underline{i}} \underline{m} D_{a} V_{\underline{m} \mid \underline{j})}\right), \\
& \hat{Q}_{\underline{i} \underline{j}, a}=V_{[\underline{\underline{i}}} \underline{\underline{m}} D_{a} V_{\underline{m} \mid \underline{j}]} .
\end{aligned}
$$

Let us finally introduce the definitions

$$
\begin{aligned}
P_{i j, \hat{D}} & \equiv M^{m}{ }_{n} V_{\hat{D}}^{D} V_{m(i} V_{j)}{ }^{n}, \quad\left(P_{i j, \hat{D}} \eta^{i j}=0\right), \\
Q_{i j, \hat{D}} & \equiv M^{m}{ }_{n} V_{\hat{D}}^{D} V_{m[i} V_{j]}{ }^{n}
\end{aligned}
$$

From (3.33) and (3.7) it is now immediate to write down the torsionfull spin connection, whose non-zero components read

$$
\begin{aligned}
\Omega_{a b, c} & =\omega_{a b, c}=e^{-\frac{\sigma}{2-D}}\left(\bar{\omega}_{a b, c}+\frac{2}{2-D} \partial_{[a} \sigma \eta_{b] c}\right), \\
\Omega_{a b, \underline{i}} & =e^{-\frac{2 \sigma}{2-D}} F_{a b}^{\frac{m}{a b}} V_{\underline{m i}}, \\
\Omega_{a \underline{i}, b} & =\Omega_{a b, \underline{i}}, \\
\Omega_{a \underline{i}, \underline{j}} & =e^{-\frac{\sigma}{2-D}}\left(\hat{P}_{\underline{i j, a}}+\frac{1}{n} \eta_{\underline{i} \underline{j}} \partial_{a} \sigma\right), \\
\Omega_{\underline{i} \underline{j}, a} & =-e^{-\frac{\sigma}{2-D}} \hat{Q}_{i j, a}, \\
\Omega_{\hat{D} i, j} & =-P_{i j, \hat{D}}, \\
\Omega_{i j, \hat{D}} & =-Q_{i j, \hat{D}} .
\end{aligned}
$$

Eq. (3.40), for $\Phi_{n}^{m} \in \mathfrak{s l}(n-1)$, may be compared with eq. (35) in [1] with a perfect agreement. We have then shown that the dimensional reduction of gravity in the presence of a torsion background of the form (3.25) is completely equivalent to a Scherk-Schwarz model with phase $M \in \mathfrak{s l}(n-1) \subset \mathfrak{e}_{6(6)}$.

Equation (3.40) contains all the ingredients to write down the $D$-dimensional Lagrangian in the presence of the torsion background, as we are going to see in section 3.3. The component $\Omega_{i j}$ does not appear in the $D$-dimensional gravity Lagrangian. However, if we consider this model as part of the bosonic sector of a supergravity theory in $D+n$ dimensions, it plays a role in the supersymmetrization of the model, since it contributes to the covariant derivative of fermion fields (it is in fact the gauged R-symmetry connection of the $(D+1)$-dimensional theory). Its components $\Omega_{i j, \hat{D}}$ give a mass to the gravitino and then it is responsible for the supersymmetry breaking. 


\subsection{The $D$ dimensional Lagrangian in the presence of torsion.}

The $D$-dimensional gravity Lagrangian is given in terms of the Einstein term modified by the presence of torsion. With our conventions we have

$$
\mathcal{L}=-2 \tilde{R} \sqrt{g_{D}} d^{D} x \equiv-2 \tilde{R}^{A B}{ }_{M N} V_{A}{ }^{[M} V_{B}{ }^{N]} \sqrt{g_{D}} d^{D} x,
$$

which is expressed in terms of

$$
\tilde{R}=\partial_{A} \Omega_{\mid B}^{A B}+\frac{1}{2} \Omega_{\mid A}^{A C} \Omega_{C B}^{\mid B}-\frac{1}{2} \Omega^{A B \mid C} \Omega_{B C \mid A} .
$$

Let us introduce

$$
\operatorname{Im} \mathcal{N}_{\underline{m n}}=-e^{-\frac{2 \sigma}{2-D}} G_{\underline{m n}},
$$

which defines the kinetic coupling of the gauge field-strengths and

$$
\mathfrak{V}=e^{\frac{2 \sigma}{2-D}} P_{i j, \hat{D}} P^{i j, \hat{D}} \geq 0,
$$

for the scalar potential. It can be verified, from inspection of the above formula, that the potential can have an absolute minimum only if $M^{m}{ }_{n} \in \mathfrak{s o}(n-1)$.

From (3.40) we find the $D$-dimensional lagrangian

$$
\begin{aligned}
\mathcal{L}=\sqrt{g_{D}} d^{D} x[ & -2 \bar{R}-\left(\frac{D}{(2-D)^{2}}+\frac{1}{n}\right) \partial_{a} \sigma \partial^{a} \sigma-\left(\hat{P}_{\underline{i} \underline{j} \mid a}-\frac{1}{n} \eta_{\underline{i} \underline{j}} \hat{P}_{\underline{k}}^{\underline{k}} \mid a\right)^{2}+ \\
& \left.+\operatorname{Im} \mathcal{N}_{\underline{m n}} F_{a b}^{\underline{m}} F^{\underline{n}} \mid a b-\mathfrak{V}\right],
\end{aligned}
$$

where $\bar{R}$ is the $D$-dimensional curvature scalar.

As we are going to see in the next section, in order to obtain the complete Lagrangian of S-S we have to fix $D=4, n=7$, and to supplement the torsion flux with non trivial fluxes for the four-form field-strength ${ }^{3}$.

\section{Other couplings in maximal S-S supergravity from internal torsion.}

So far, we have been considering the effect of an internal torsion background on the dimensional reduction of the $(D+n)$-dimensional metric.

Let us now look at this from a slightly different point of view, in the spirit of the toy model of Section 2. In particular, let us study the coupling of a gauge field $A_{M}$ to an internal torsion of the form (3.25). As discussed in section 3.2, the presence of torsion will contribute with a term of the form

$$
F_{D m}=\frac{1}{2} M_{m}^{n} A_{n}
$$

The same contribution to the field strength could be obtained in the absence of torsion by assuming an effective dependence of the vector $A_{M}$ on the coordinate $X^{D}=y^{1}$, of the form (in matrix notation)

$$
\tilde{A}(x, y)=U\left(y^{1}\right) \cdot A(x),
$$

\footnotetext{
${ }^{3}$ Evidence for completion of the S-S phase with the 4-form flux may also be found in Ref. [15]
} 
with $U\left(y^{1}\right)$ given by

$$
U\left(y^{1}\right):\left(\begin{array}{ccc}
\delta^{\mu} & 0 & 0 \\
0 & 1 & 0 \\
0 & 0 & \left(e^{\Phi}\right)_{n}^{m}
\end{array}\right), \quad \Phi_{n}^{m}=y^{1} M_{n}^{m},
$$

since it would give a contribution

$$
F_{D n}=\frac{1}{2} \partial_{D} A(x, y)_{n}=\frac{1}{2} M_{n}^{m} A_{m} .
$$

The gauge transformations that preserve the form (4.2) of the gauge field are of the generalized class (2.3), with the tensor $C_{N}^{M}=U\left(y^{1}\right)_{N}^{M}$, even if, from the $D$ dimensional point of view, the gauge transformation of the vector $\tilde{A}_{\mu}=A_{\mu}$ is the usual one,

$$
A_{\mu} \rightarrow A_{\mu}+\partial_{\mu} \Lambda(x)
$$

This is in fact the case for any tensor $C_{N}^{M}$ which has non-trivial entries in the components with upper index $M \neq \mu$. The matrix $U\left(y^{1}\right)$ is precisely of this form.

The same point of view may be applied to another field with spin, the vielbein. We have found in section 3.2 that the effect of an internal torsion background (3.25) is to induce non abelian couplings for the four dimensional gauge vectors and scalars coming from the metric, as in (3.40). Just as above, we observe that the same result (3.40) might have been obtained in the presence of a torsionless spin connection, if we had assumed for the $(D+n)$-dimensional vielbein an effective dependence on the internal coordinate $X^{D}=y^{1}$, of the form

$$
\tilde{\mathcal{V}}_{M}^{A}(x, y)=U\left(y^{1}\right)_{M}^{N} \mathcal{V}_{N}^{A}(x),
$$

with $U\left(y^{1}\right)$ given by (4.3), which is precisely the spirit of Scherk-Schwarz dimensional reduction (for an $X^{D}$-dependent $\mathrm{SL}(6)$ phase).

We observe that we can now then reinterpret the S-S phase as the back-reaction on the $(D+n)$-dimensional space-time geometry of the presence of the torsion flux. ${ }^{4}$

The interplay between generalized gauge invariance in the presence of internal torsion and S-S phases may be further studied by considering generalized gauge invariance, in the presence of torsion, for p-forms. Let us take, for the remaining part of this section, $D=4$, $n=7$, and consider the case of the bosonic sector of eleven dimensional supergravity. Besides the metric $g_{M N}$, this theory contains a three-form $A_{M N P}$. In the presence of a torsion of type (3.25), its electric field strength $F_{M N P Q}$ gets a non vanishing contribution due to the torsion, namely

$$
F_{D m n p}=\frac{1}{4} M_{[m}^{q} A_{n p] q},
$$

with $M=\partial_{D} \Phi \in \mathrm{SL}(6, \mathbb{R})$. It would then appear, in the four dimensional theory, as an effective flux, induced by the presence of torsion.

${ }^{4}$ We thank J.F. Morales for an enlightening discussion on this point. 
We have seen that the effect of an internal torsion on spinfull fields may be equivalently taken into account by multiplying the corresponding gauge field by an appropriate matrix $U_{n}^{m}(y)$ such that $T_{4 n}^{m}=\left(U^{-1}\right)_{p}^{m} \partial_{y} U_{n}^{p}$. This can be understood as a consequence of the presence of torsion, which forces the generalized rule (2.3) for gauge invariance. In a general gauge the $(D+1)$ dimensional gauge potential, corresponding to the zero-mode in the K-K expansion, has effectively a dependence on the coordinate $y$. This leads to the identification of the tensor $C$, needed to re-establish the gauge invariance, with the S-S phase $U$ as in (4.4).

However, when considering gauge potentials corresponding to p-forms, the generalized gauge invariance of the four dimensional reduced theory (further restricted by the request of having also a five dimensional interpretation) still leaves room for introducing a more general tensor $C$ like

$$
\delta A_{M_{1} \ldots M_{p}}=C_{M_{1} \ldots M_{p}}^{N_{1} \ldots N_{q}}(y) \partial_{\left[N_{1}\right.} \Lambda_{\left.N_{2} \ldots N_{q}\right]}(x),
$$

having non trivial entries only in the internal directions.

This corresponds to a freedom left to choose the fluxes in a more general way than (4.5). As we are going to see in the next section, they will complete the phase (4.3) to generate a phase in the adjoint representation $\mathbf{7 8}$ of $\mathfrak{e}_{6(6)}$. Here, appropriate fluxes, which include a generalization of (4.5), provide the components needed to complete an $\mathfrak{e}_{6(6)}$ phase from the one in $\mathfrak{s l}(6, \mathbb{R})$. The analysis will be performed with a solvable algebra approach, in order to make a precise identification of the four-dimensional fields in terms of eleven-dimensional degrees of freedom.

This will complete the proof of the equivalence of the S-S model with a flux plus torsion compactification.

\section{Completion of the S-S phase}

Consider the four dimensional maximal supergravity obtained through a S-S reduction from $D=5$. As anticipated in the introduction, the five dimensional Lagrangian has an $\mathrm{E}_{6(6)}$ global symmetry. If this theory is thought of as originating from a toroidal compactification of eleven dimensional supergravity, then all its fields transform manifestly with respect to the subgroup $\mathrm{SL}(6, \mathbb{R}) \times \mathrm{SL}(2, \mathbb{R}) \subset \mathrm{E}_{6(6)}$, where $\mathrm{SL}(6, \mathbb{R})$ is the group acting on the metric moduli (except the volume) of the internal torus $T^{6}$, while the dilation modulus of the $T^{6}$ volume, $\hat{\sigma}$, is contained inside $\operatorname{SL}(2, \mathbb{R})$. The five dimensional scalars originating from the eleven dimensional fields $G_{M N}$ and $A_{M N P}$ are:

$$
G_{m n}, \quad A_{m n p}, \quad \tilde{A}
$$

where $m, n, p=5, \ldots, 10 ;^{5} M, N, P=0, \ldots, 10 . \tilde{A}$ denotes the scalar dual to $A_{\hat{\mu} \hat{\nu} \hat{\rho}}$ $(\hat{\mu}, \hat{\nu}, \hat{\rho}=0, \ldots, 4)$. All together, they span the coset manifold $\mathcal{M}_{5}=\mathrm{E}_{6(6)} / \mathrm{USp}(8)$, of dimension $42=21+20+1$. $\mathcal{M}_{5}$ can be thought of as parametrized by the scalar fields

\footnotetext{
${ }^{5}$ For later convenience we adopt here a slight change of notation, and the indices in the internal dimensions will now run from 5 to 10 .
} 
listed above, and it has the structure of a solvable Lie group, inherited from the Iwasawa decomposition of the non compact group $\mathrm{E}_{6(6)}$. We will denote by $\mathfrak{s o l v}_{5}$ the solvable Lie algebra associated to $\mathcal{M}_{5}$ [26]. It is a Borel subalgebra consisting of the non compact Cartan elements (in this case all the Cartan elements can be chosen non compact) semidirect product with a nilpotent algebra formed by the shift generators associated to the positive (restricted) roots. With respect to the subgroup $\mathrm{SL}(6, \mathbb{R}) \times \mathrm{SL}(2, \mathbb{R})$, the adjoint representation (78) of $\mathrm{E}_{6(6)}$ branches as follows:

$$
\mathbf{7 8} \underset{\mathrm{SL}(6, \mathbb{R}) \times \mathrm{SL}(2, \mathbb{R})}{\longrightarrow}(35, \mathbf{1})+(\mathbf{1}, \mathbf{3})+(\mathbf{2 0}, \mathbf{2}),
$$

being 35 the adjoint representation of $\mathrm{SL}(6, \mathbb{R})$. Let us denote by $t^{n}{ }_{m}$ the $\mathfrak{s l}(6, \mathbb{R})$ generators in the $(\mathbf{3 5}, \mathbf{1}), t^{\alpha m n p}$ the nilpotent generators in the $(\mathbf{2 0 , 2})(\alpha=1,2$ being the doublet index) and by $s^{\alpha}{ }_{\beta}$ the $\mathfrak{s l}(2, \mathbb{R})$ generators in the $(\mathbf{1}, \mathbf{3})$. A generic $\mathfrak{e}_{6(6)}$ generator $M$ will be a linear combination

$$
M=\theta_{m}^{n} t_{n}^{m}+\xi_{\alpha}^{\beta} s_{\beta}^{\alpha}+\theta_{\alpha m n p} t^{\alpha m n p} .
$$

The solvable algebra $\mathfrak{s o l v} \mathfrak{v}_{5}$ is generated by $t^{n}{ }_{m}(m \geq n), t^{1 m n p}$ and $s^{\alpha}{ }_{\beta}(\beta \geq \alpha)$. We can choose a coset representative $\mathbb{L} \in \mathrm{E}_{6(6)}$ of $\mathcal{M}_{5}$ as

$$
\mathbb{L}=\exp \left(A_{m n p} t^{1 m n p}\right) \exp \left(\tilde{A} s_{2}{ }_{2}\right) \exp \left(\sum_{m \geq n} \gamma^{m}{ }_{n} t^{n}{ }_{m}\right) \exp \left(\hat{\sigma} t^{1}{ }_{1}\right)
$$

$\gamma^{m}{ }_{n}$ being the infinitesimal moduli defining the internal metric (with volume normalized to 1 ). In fact, the vielbein of the internal 6-torus, $V^{i}=V_{m}^{i} d x^{m}$, is a coset representative of $\mathrm{GL}(6) / \mathrm{SO}(6)$, and we have

$$
\left.V=\exp \left(\sum_{m \geq n} \gamma^{m}{ }_{n} t^{n}{ }_{m}\right)\right) \exp \left(\hat{\sigma} t^{1}{ }_{1}\right)
$$

The eleven dimensional origin of the fields appearing in the solvable parametrization of the scalar manifold in 5 dimensions was disclosed in Ref. [26].

Consider now taking $M$ in (5.3) as a S-S generator for a S-S dimensional reduction to $D=4$. Let us denote by $x=\left(x^{\mu}\right)$ the four dimensional coordinates and by $y=X^{4}$ the fifth direction. Let $S$ be a generic scalar field of the set $\left\{\gamma_{m n}, \hat{\sigma}, A_{m n p}, \tilde{A}\right\}$. Then we have

$$
\mathbb{L}[S(x, y)]=U(y) \mathbb{L}[S(x)] h[S(x), U],
$$

where the compensator $h$, which is an element of $\operatorname{USp}(8)$, has to be introduced to keep the form (5.4). Let $M$ be a generator of $\mathfrak{e}_{6(6)}$ of the form (5.3). Since it belongs to the isometry algebra of $\mathcal{M}_{5}$, it has associated a Killing vector denoted by $k_{(M)}$. In terms of the solvable parametrization, it can be written as

$$
k_{(M)}=k_{(M)}^{S} \partial_{S}
$$

A generic scalar field $S$ in the S-S ansatz is thus expressed in the following way:

$$
S(x, y)=S(x)+\delta S(x, y)=S(x)+y k_{(M)}^{S}+O\left(y^{2}\right)
$$


The contributions to the Lagrangian will appear in the sigma model term for the scalars,

$$
\left\langle\left(\mathbb{L}^{-1} d L\right)_{\mathrm{nc}},\left(\mathbb{L}^{-1} d L\right)_{\mathrm{nc}}\right\rangle,
$$

("nc" stands for the projection on the non compact part). One can convince himself that the only possible contributions come for the first order term in $y$, the one proportional to the Killing vector himself in (5.8). This is because $k_{(M)}$ is a global symmetry of the Lagrangian.

The key point in order to interpret these parameters in terms of background fluxes/torsion, is to control the higher dimensional origin of each scalar field. This is possible thanks to the solvable Lie algebra parametrization of the scalar manifold which we have adopted. Indeed, the correspondence to be considered is:

$$
\text { background fluxes/torsion } \equiv \partial_{y} S(x, y)_{\mid y=0}=k_{(M)}(x),
$$

being $k_{(M)}$ isometries that could in principle be gauged by the vectors in the theory.

Let us consider a S-S generator (5.3) with only the parameters $\theta^{n}{ }_{m} \neq 0$, and let us consider a particular $\mathbb{L}$ with $A_{m n p}=\hat{A}=0$. Then the internal vielbein introduced in (4.4) reads, in this formalism, as:

$$
V_{m}^{i}(x, y)=[U(y) \mathbb{L}(\gamma(x), \hat{\sigma}(x))]_{m}^{i} .
$$

The parameters $\theta^{n}{ }_{m}$ will enter the lower dimensional Lagrangian through the quantity:

$$
T_{D m}^{n}=V_{i}^{n}(x, y) \partial_{y} V_{m}{ }^{i}(x, y)_{\mid y=0}=\theta_{m}^{n},
$$

which coincides with the internal torsion introduced in section 3.

Similarly we can switch on only the scalar fields $A_{m n p}, \tilde{A}$ and take a S-S phase with parameters $\theta_{1 m n p}, \theta_{m}^{n}$ and $\xi^{2}$. We have:

$$
\begin{aligned}
A_{m n p}(x, y) & =A_{m n p}(x)+y \theta_{1 m n p}+y \theta_{[m}^{r} A_{n p] r}(x)+O\left(y^{2}\right), \\
\tilde{A}(x, y) & =\tilde{A}(x)+y \xi^{2}{ }_{1} .
\end{aligned}
$$

Using eq. (5.10) we can write the following correspondence between M-theory fluxes and S-S parameters, which generalizes (4.5):

$$
\begin{aligned}
F_{y m n p} & =\partial_{y} A_{m n p}(x, y)_{\mid y=0}=\theta_{1 m n p}+\theta_{[m}^{r} A_{n p] r}(x), \\
\frac{1}{6 !} \epsilon^{m_{1} \ldots m_{6}} F_{y m_{1} \ldots m_{6}} & =\partial_{y} \tilde{A}(x, y)_{\mid y=0}=\xi^{2}{ }_{1} .
\end{aligned}
$$

The second term on the right hand side of eq. (5.14) is required by the definition of field strength in the presence of internal torsion. So far we have been considering the microscopic interpretation of the S-S parameters corresponding to the choice of $\mathrm{M}$ either inside the full $\mathfrak{s l}(6, \mathbb{R})$ or in the remaining part of the $\mathfrak{e}_{6(6)}$ Borel subalgebra, namely among the nilpotent generators $t^{1 m n p}, s^{1}{ }_{2}$. Switching on $\theta_{2 m n p}$ and $\xi^{2}{ }_{1}$ would introduce in the Killing vector $k_{(M)}$, and thus on the right hand side of eqs. (5.14), (5.15), more involved scalar-dependent terms. Nevertheless, also in this case, the general eq. (5.10), plus the microscopic interpretation of the scalar fields $S$, would provide the relation between fluxes and S-S parameters. This is work in progress, which will be presented elsewhere. 


\section{A four dimensional analysis}

In this section we shall adopt a four dimensional viewpoint and analyze the gauging of maximal supergravity originating from a S-S reduction using a group theoretical framework which allows to interpret the parameters in terms of ten or eleven dimensional quantities. This mathematical method will also prove particularly useful in the study of the action of dualities (e.g. T-duality) on the fields or background flux/torsion.

The field equations and Bianchi identities of $D=4, N=8$ supergravity are invariant under the global symmetry group $\mathrm{E}_{7(7)}[27]$, which is the isometry group of the scalar manifold $\mathcal{M}_{4}=\mathrm{E}_{7(7)} / \mathrm{SU}(8)$ spanned by the scalars of the theory. The electric and magnetic charges $Q_{I}(I=1, \ldots, 56)$ transform in the representation $\mathbf{5 6}$ of $\mathrm{E}_{7(7)}$. Gauging the theory means to promote a suitable global symmetry group $\mathcal{G} \subset \mathrm{E}_{7(7)}$ of the Lagrangian to be a local symmetry. To this end, besides introducing minimal couplings which involve the vector fields of the model, the Lagrangian should be further deformed by the addition of fermionic mass terms and a scalar potential, which are required by consistency of the new local symmetry with $N=8$ supersymmetry.

Let $\left\{t_{\ell}\right\}_{\ell=1, \cdots, 133}$ be a basis of $\mathfrak{e}_{7(7)}$. The most general gauging of this theory is defined by an embedding tensor $\Theta_{I}^{\ell}$ [24]. This tensor defines the embedding of the gauge group $\mathcal{G}$ inside $\mathrm{E}_{7(7)}$, since it expresses the generators $\left\{T_{I}\right\}$ as combinations of the $\mathfrak{e}_{7(7)}$ generators $t_{\ell}$ :

$$
T_{I}=\Theta_{I}^{\ell} t_{\ell}
$$

In this formulation the 28 vector fields of the theory are labelled by a subset of values of $I$, and since any gauging can involve only elementary vector fields and not their magnetic duals, $\Theta$ has necessarily rank $r \leq 28$, so that the $T_{I}$ may be interpreted as gauge generators. The gauged field equations and Bianchi identities are not $\mathrm{E}_{7(7)}$-invariant anymore, since the original duality symmetry is explicitlytly broken by $\Theta$. This global symmetry can be formally restored if $\Theta$ is thought of as an $E_{7(7)}$-covariant tensor in the $\mathbf{5 6} \times \mathbf{1 3 3}$ and thus made to transform under the original duality symmetry as well. Supersymmetry further constrains $\Theta$ to transform in the $\mathbf{9 1 2}$ of $\mathrm{E}_{7(7)}$ contained in $\mathbf{5 6} \times \mathbf{1 3 3}$ [24]. However different choices of $\Theta$ define different theories and therefore a transformation of $\Theta$ should not be understood as a symmetry of the theory but rather as a duality mapping between different gauged models. Since compactifications in the presence of internal fluxes and/or torsion (in the limit in which these background v.e.v. are "small enough") typically give rise to a gauged lower-dimensional supergravity, this mathematical approach is particularly suitable for the study of dualities between different flux/torsion vacua.

In all known gauged supergravities originating from flux compactifications, the background fluxes enter the low energy gauged supergravity through the embedding tensor of the gauge group [10]. Our previous analysis, which identifies components of an internal torsion as coupling constants in the corresponding S-S gauged supergravity, provides strong evidence in favor of the following correspondence:

$$
\text { Background fluxes/torsion } \equiv \Theta_{I}^{\ell} \text {. }
$$


The above identification tells us that background fluxes and torsion are part of an $\mathrm{E}_{7(7)}$ representation (namely the $\mathbf{9 1 2}$ ) and thus can be associated with suitable weights. In what follows we shall study this correspondence for the background quantities which are relevant to the S-S supergravity.

The higher dimensional origin of the four dimensional scalar fields, vectors and coupling constants can be understood by branching the adjoint representation of $\mathrm{E}_{7(7)}{ }^{6}$, the 56 and the 912 respectively, with respect to some maximal subgroup of $\mathrm{E}_{7(7)}$ which characterizes the dimensional reduction. Indeed, if the four dimensional theory originates from dimensional reduction of the five dimensional maximal supergravity, then the relevant subgroup of $\mathrm{E}_{7(7)}$ is $\mathrm{E}_{6(6)} \times \mathrm{SO}(1,1)$ and we have $[7,24]$ :

$$
\begin{aligned}
& \mathfrak{s o l v} \mathfrak{v}_{4} \longrightarrow \mathfrak{s o l v}_{5}+\mathfrak{s o}(1,1)+\overline{\mathbf{2 7}}_{+2}, \\
& \mathbf{5 6} \longrightarrow \overline{\mathbf{2 7}}_{-1}+\mathbf{1}_{-3}+\mathbf{2 7 _ { + 1 }}+\mathbf{1}_{+3}, \\
& \mathbf{9 1 2} \longrightarrow \overline{\mathbf{3 5 1}}_{-1}+\mathbf{3 5 1 _ { + 1 }}+\overline{\mathbf{2 7}}_{-1}+\mathbf{2 7 _ { + 1 }}+\mathbf{7 8 _ { + 3 }}+\mathbf{7 8 _ { - 3 }} \text {, }
\end{aligned}
$$

where in the first branching $\mathfrak{s o l v} \mathfrak{v}_{5}$ is parametrized by the five dimensional scalar fields, $\mathfrak{s o}(1,1)$ by the radius of the fifth dimension and $\overline{\mathbf{2 7}}_{+2}$ by the axions $A_{4}^{\Lambda}$ originating from the 27 five dimensional vector fields $A_{\hat{\mu}}^{\Lambda}(\Lambda=1, \ldots, 27)$. In the branching of the $\mathbf{5 6}$, the $\overline{\mathbf{2 7}}_{-1}$ and $\mathbf{1}_{-3}$ represent the vectors $A_{\mu}^{\Lambda}$ coming from the five dimensional vectors and the Kaluza-Klein vector $B_{\mu}$, while the remaining representations correspond to their magnetic duals. Each representation in the branching of the $\mathbf{9 1 2}$ describes a different gauging of the four dimensional theory [24]. In particular the $\mathbf{7 8}_{+3}$ defines the S-S gauging. Indeed the corresponding embedding matrix $\Theta$ can be expressed in terms of an element $M_{\Lambda}{ }^{\Sigma}$ of $\mathbf{7 8}$ (the S-S generator) with grading +3 with respect to $\mathrm{SO}(1,1)$ and the gauge generators are given, from (6.1), by:

$$
T_{0}=M_{\Lambda}{ }^{\Sigma} t_{\Sigma}^{\Lambda} ; T_{\Lambda}=M_{\Lambda}{ }^{\Sigma} t_{\Sigma},
$$

where $t_{\Sigma}{ }^{\Lambda}$ form a basis of $\mathfrak{e}_{6(6)}$ while $t_{\Sigma}$ are the $\mathfrak{e}_{7(7)}$ generators in the $\overline{\mathbf{2 7}}_{+2}$ of $\mathrm{E}_{6(6)}$. They thus close the algebra $[1,7]$

$$
\left[T_{0}, T_{\Lambda}\right]=M_{\Lambda}{ }^{\Sigma} T_{\Sigma} ; \quad\left[T_{\Lambda}, T_{\Sigma}\right]=0 .
$$

If the four dimensional theory is interpreted, along the lines of the analysis followed throughout our paper, as resulting from a two-step dimensional reduction $D=11 \rightarrow D=5 \rightarrow$ $D=4$, then we should consider branchings with respect to the following subgroups of $\mathrm{E}_{7(7)}$ :

$$
\mathrm{SL}(6, \mathbb{R}) \times \mathrm{SL}(2, \mathbb{R}) \times \mathrm{O}(1,1) \subset \mathrm{E}_{6(6)} \times \mathrm{SO}(1,1) \subset \mathrm{E}_{7(7)}
$$

With respect to $\mathrm{SL}(6, \mathbb{R}) \times \mathrm{SL}(2, \mathbb{R}) \times \mathrm{SO}(1,1)$ the $\mathrm{S}-\mathrm{S}$ embedding tensor in the $\mathbf{7 8}_{+3}$ branches as in (5.2), namely

$$
\mathbf{7 8} 8_{+3} \rightarrow(35,1)_{+3}+(\mathbf{1}, 3)_{+3}+(\mathbf{2 0}, \mathbf{2})_{+3} .
$$

\footnotetext{
${ }^{6}$ The scalar fields parametrize the manifold $\mathcal{M}_{4}$, a solvable group with Lie algebra $\mathfrak{s o l v}_{4} \subset \mathfrak{e}_{7(7)}$
} 
Note that all the representations on the right hand side have $\mathrm{SO}(1,1)$-grading +3 and this is a stringent condition in identifying the corresponding fluxes/torsion.

In order to associate explicit $\mathfrak{e}_{7(7)}$ weights to the various fields and background fluxes and torsion, let us now consider the four dimensional theory as deriving from a Type II theory in $D=10$.

We shall express the $\mathfrak{e}_{7(7)}$ weights in terms of a certain orthonormal basis Cartan subalgebra $\left\{\epsilon_{\underline{n}}\right\}^{7}$

$$
\epsilon_{\underline{n}} \cdot \epsilon_{\underline{m}}=\delta_{\underline{n m}}, \quad \underline{n}=4, \ldots 10 .
$$

This basis is chosen in such a way that the positive simple roots $\alpha_{1}, \ldots \alpha_{7}$ are expressed as

$$
\begin{aligned}
& \alpha_{\underline{n}-3}=\epsilon_{\underline{n}}-\epsilon_{\underline{n}+1} \quad(\text { for } \underline{n}=4, \ldots, 8) ; \\
& \alpha_{6}=\epsilon_{8}+\epsilon_{9} ; \\
& \alpha_{7}=a \quad \text { (IIB); } \quad \alpha_{7}=a+\epsilon_{9} \quad \text { (IIA) }
\end{aligned}
$$

with $a$ defined as

$$
a=-\frac{1}{2} \sum_{r=4}^{9} \epsilon_{r}+\frac{1}{\sqrt{2}} \epsilon_{10} .
$$

The two choices (IIA) and (IIB) give isomorphic algebras $\mathfrak{e}_{7,7}$, and they will be justified by the interpretation of the fields in the higher dimensional theory.

The scalar fields parametrize the solvable group $\mathcal{M}_{4}$, whose Lie algebra $\mathfrak{s o l v}_{4}$ is generated by the seven (non compact) Cartan generators of $\mathfrak{e}_{7(7)}$ (corresponding to the moduli $\sigma_{r}$ of the six internal radii $R_{r}, r=4, \ldots, 9$, and the dilaton $\phi$ ), and by 63 shift generators associated with the positive roots and corresponding to the axionic scalar fields. In the basis $\left\{\epsilon_{\underline{n}}\right\}$ the correspondence between axionic scalar fields and positive roots reads [24]:

$$
\begin{aligned}
A_{r_{1} r_{2} \ldots r_{k}} & \leftrightarrow a+\epsilon_{r_{1}}+\ldots \epsilon_{r_{k}}, \\
\tilde{A}_{r_{1} r_{2} \ldots r_{k} \mu \nu} & \leftrightarrow a+\epsilon_{s_{1}}+\ldots \epsilon_{s_{6-k}}, \quad\left(\epsilon^{r_{1} \ldots r_{k} s_{1} \ldots s_{6-k}} \neq 0\right), \\
B_{r s} & \leftrightarrow \epsilon_{r}+\epsilon_{s}, \\
\tilde{B}_{\mu \nu} & \leftrightarrow \sqrt{2} \epsilon_{10}, \\
\gamma_{s}^{r} & \leftrightarrow \epsilon_{s}-\epsilon_{r}, \quad(r>s),
\end{aligned}
$$

By $A$ we have denoted generically the RR ten dimensional forms in either Type IIA ( $k$ even) or Type IIB ( $k$ odd) theories, and the corresponding choices (6.9) must be understood; $B$ denotes the ten dimensional Kalb-Ramond field and $\gamma$, as usual, are the moduli of

\footnotetext{
${ }^{7}$ For the sake of clarity let us summarize the conventions on internal indices which will be followed in the present section. Unless explicitly stated, we shall assume $M, N=0, \ldots, 10$ (labelling the eleven dimensional space-time); $\underline{n}, \underline{m}=4, \ldots, 10$ (the internal indices when reducing from $D=11$ to $D=4$, used in view of the interpretation of $D=4$ quantities in terms of M-theory); $n, m=5, \ldots, 10$ (the internal indices when reducing from $D=11$ to $D=5$, used in view of the interpretation of $D=5$ quantities in terms of M-theory); $r, s=4, \ldots, 9$ (the internal indices when reducing from $D=10$ to $D=4$, used in view of type IIA/IIB interpretations of $D=4$ quantities); $u, v=5, \ldots, 9$ (the internal indices when reducing from $D=10$ to $D=5$, used in view of type IIA/IIB interpretations of $D=5$ quantities).
} 
the internal metric. The symbols with a tilde " " denote the scalars dual to the four dimensional 2-forms.

For the vectors and their corresponding duals, since they are in the representation $\mathbf{5 6}$, they must be in one-to-one correspondence with the weights $W$ of the $\mathbf{5 6}$ of $\mathrm{E}_{7(7)}$ :

$$
\begin{aligned}
A_{r_{1} \ldots r_{k} \mu} & \leftrightarrow w+\epsilon_{r_{1}}+\ldots \epsilon_{r_{k}} \\
B_{r \nu} & \leftrightarrow \epsilon_{r}-\frac{1}{\sqrt{2}} \epsilon_{10} \\
\gamma_{\mu}^{r} & \leftrightarrow-\epsilon_{r}-\frac{1}{\sqrt{2}} \epsilon_{10}
\end{aligned}
$$

where

$$
w=-\frac{1}{2} \sum_{r=4}^{9} \epsilon_{r},
$$

and the opportune choice (6.9) must be understood. The dual potentials correspond to the opposite weights $-W$.

The above field-weight correspondence can be deduced by considering the kinetic terms in the Lagrangian of the various fields as they arise from a reduction of the ten dimensional theory on a straight torus. They have the form:

$$
\begin{aligned}
\text { dilatonic scalars: } & -\partial_{\mu} h \cdot \partial^{\mu} h, \\
\text { axionic scalars: } & -\frac{1}{2} e^{-2 \alpha \cdot h}\left(\partial_{\mu} \varphi \cdot \partial^{\mu} \varphi\right), \\
\text { vector fields: } & -\frac{1}{4} e^{-2 W \cdot h} F_{\mu \nu} F^{\mu \nu},
\end{aligned}
$$

where

$$
h=h(\sigma, \phi)=\sum_{r=4}^{9} \sigma_{r} \epsilon_{r}-\sqrt{2} \phi_{4} \epsilon_{10},
$$

and $\alpha$ and $W$ are the positive root and the weight associated with the generic axion $\varphi$ and vector field $A_{\mu}$ respectively.

For the internal metric (in the ten dimensional string frame) we have chosen $G_{r s}=$ $e^{2 \sigma_{r}} \delta_{r s}$ and $\phi_{4}$ is the four dimensional dilaton, which is related to the ten dimensional one by

$$
\phi_{4}=\phi-\frac{1}{2} \sum_{r=4}^{9} \sigma_{r} .
$$

To understand the field-weight correspondence, consider for instance the kinetic term of $A_{r_{1} r_{2} \ldots r_{k}}$; it reads:

$$
\begin{aligned}
& \sqrt{\operatorname{det} G_{(10)}} G_{(10)}^{\mu \nu} G_{(10)}^{r_{1} r_{1}} \ldots G_{(10)}^{r_{k} r_{k}} \partial_{\mu} A_{r_{1} r_{2} \ldots r_{k}} \partial_{\nu} A_{r_{1} r_{2} \ldots r_{k}}= \\
& \left(\sqrt{\operatorname{det} g_{(4)}} e^{\sum_{r=4}^{9} \sigma_{r}+4 \phi_{4}}\right) e^{-2 \phi_{4}-2 \sum_{i=1}^{k} \sigma_{r_{i}}} \partial_{\mu} A_{r_{1} r_{2} \ldots r_{k}} \partial^{\mu} A_{r_{1} r_{2} \ldots r_{k}}= \\
& \sqrt{\operatorname{det} g_{(4)}} e^{-2 \alpha \cdot h}\left(\partial_{\mu} A_{r_{1} r_{2} \ldots r_{k}}\right)^{2},
\end{aligned}
$$


where we have used $G_{(10) \mu \nu}=e^{2 \phi_{4}} g_{(4) \mu \nu}$. Using the above recipe we can associate a weight with any flux (either of RR or NS origin) or torsion, by inspection of the corresponding quadratic term in the four dimensional Lagrangian. We thus have ${ }^{8}$

$$
\begin{aligned}
F_{\mu_{1} \ldots \mu_{p} r_{1} \ldots r_{k}} & \leftrightarrow-\frac{1}{2} \sum_{r=4}^{9} \epsilon_{r}+\sum_{i=1}^{k} \epsilon_{r_{i}}+\frac{2-p}{\sqrt{2}} \epsilon_{10}, \\
H_{r s t} & \leftrightarrow \epsilon_{r}+\epsilon_{s}+\epsilon_{t}+\frac{\epsilon_{10}}{\sqrt{2}} \\
T_{r s}^{t} & \leftrightarrow \epsilon_{r}+\epsilon_{s}-\epsilon_{t}+\frac{\epsilon_{10}}{\sqrt{2}},
\end{aligned}
$$

where $F=d A, H=d B$ are the RR and NS field-strengths and $T$ is the internal torsion.

After this formal treatment, we can now make contact with the analysis made in the previous section about S-S reduction from five dimensions. We had interpreted the five dimensional scalar fields from an 11 dimensional (M-theory) point of view. They correspond to $A_{m n p}, \tilde{A}$ and $\gamma^{n}{ }_{m}$. When we perform a reduction from 11 to 10 dimensions, and then to 4 , they can be interpreted in a Type IIA language as $A_{u v w}, B_{u v}=A_{u v 10}, \tilde{A}$, $\gamma^{u}{ }_{v}(u>v), A_{u}=\gamma^{10}{ }_{v}$, where $u, v, w=5, \ldots, 9$ and the corresponding positive roots are (6.11):

$$
\begin{aligned}
A_{u v w} & \leftrightarrow a+\epsilon_{u}+\epsilon_{v}+\epsilon_{w}, \\
\tilde{A} & \leftrightarrow a+\sum_{r=5}^{9} \epsilon_{r}, \\
A_{u} & \leftrightarrow a+\epsilon_{u}, \\
B_{u v} & \leftrightarrow \epsilon_{u}+\epsilon_{v}, \\
\gamma^{u}{ }_{v} & \leftrightarrow-\epsilon_{u}+\epsilon_{v} .
\end{aligned}
$$

The $\mathrm{SO}(1,1)$ parametrizing the radius of the fifth $\left(X^{4}\right)$ dimension is generated by the Cartan generator $H_{\lambda}$ associated to the weight $\lambda=2 \epsilon_{4}+\sqrt{2} \epsilon_{10}$ (which is the highest weight of the $\mathbf{5 6}$ of $\mathrm{E}_{7(7)}$ ). The M-theory fluxes which we have associated with (part of the) S-S parameters are $F_{4 m n p}$ and $F_{4 m_{1} \ldots m_{6}}$. They are interpreted from Type IIA point of view as the fluxes $F_{4 u v w}, H_{4 u v}=F_{4 u v} 10$ and $\tilde{F}_{\mu \nu \rho \sigma}$ and are associated with the weights:

$$
\begin{aligned}
F_{4 u v w} & \leftrightarrow-\frac{1}{2} \sum_{r=4}^{9} \epsilon_{r}+\epsilon_{4}+\epsilon_{u}+\epsilon_{v}+\epsilon_{w}+\sqrt{2} \epsilon_{10}, \\
H_{4 u v} & \leftrightarrow \epsilon_{4}+\epsilon_{u}+\epsilon_{v}+\frac{\epsilon_{10}}{\sqrt{2}}, \\
\tilde{F}_{\mu \nu \rho \sigma} & \leftrightarrow \frac{1}{2} \sum_{r=4}^{9} \epsilon_{r}+\sqrt{2} \epsilon_{10},
\end{aligned}
$$

\footnotetext{
${ }^{8} \mathrm{~A}$ similar correspondence between components of ten dimensional field strengths and weights of the lower-dimensional duality group was used in [28] in connection to the study of the cosmological billiard phenomenon.
} 
where $\tilde{F}_{\mu \nu \rho \sigma}$ is the 7 -form flux dual in eleven dimensions to $F_{\mu \nu \rho \sigma}$ and its weight is thus the opposite to the corresponding one which can be read off from eqs. (6.20). As far as the internal torsion $T_{4 n}^{m}$ is concerned, it corresponds, from the Type IIA point of view, to the ten-dimensional internal torsion $T_{4 u}^{v}$ and to a component of the RR two-form field strength, $F_{4 u}=T_{4 u}^{10}$. They are associated with the weights:

$$
\begin{aligned}
& T_{4 u}^{v} \leftrightarrow \epsilon_{4}+\epsilon_{u}-\epsilon_{v}+\frac{\epsilon_{10}}{\sqrt{2}}, \\
& F_{4 u} \leftrightarrow-\frac{1}{2} \sum_{r=4}^{9} \epsilon_{r}+\epsilon_{4}+\epsilon_{u}+\sqrt{2} \epsilon_{10} .
\end{aligned}
$$

Using the weight representation (6.22), (6.23) of the relevant fluxes and torsion involved in the S-S gauging, one may check that their identification with components of the S-S embedding tensor in the $\mathbf{7 8}_{+3}$ is indeed consistent. Here we shall only give some evidence based on grading arguments. The $\mathrm{SO}(1,1)$ weight associated with the above fluxes is given by the scalar product of $\lambda$ times the related weight. As it can be easily verified this product gives +3 for all the above fluxes. Indeed these weights can all be expressed as

$$
W_{(\mathrm{S}-\mathrm{S} \text { flux } / \text { torsion })}=\lambda / 2+\beta
$$

where $\beta$ is a root of $\mathfrak{e}_{6(6)}$ such that $\lambda \cdot \beta=0$.

This is consistent with our interpretation of these flux/torsion components as belonging to the $\mathbf{7 8}+3$.

Let us now comment on the transformation properties of the fluxes/torsion in (6.22), (6.23) with respect to the $\mathrm{SL}(2, \mathbb{R})$ group in (6.6). We find that the fluxes $F_{4 u v w}, H_{4 u v}$ belong to a doublet (the $(\mathbf{2 0 , 2})_{+3}$ in $\left.(6.7)\right), \tilde{F}_{\mu \nu \rho \sigma}$ to a triplet (the $(\mathbf{1}, \mathbf{3})_{+3}$ in (6.7)) while $T_{4 u}^{v}(v>u), F_{4 u}$ are singlets (in the $(\mathbf{3 5}, \mathbf{1})_{+3}$ in $\left.(6.7)\right)$. To show this it suffices to check the grading relative to the Cartan generator $H_{\tilde{\alpha}}$ of the corresponding $\mathfrak{s l}(2, \mathbb{R})$ algebra. The positive root $\tilde{\alpha}$ of $\mathfrak{s l}(2, \mathbb{R})$ is

$$
\tilde{\alpha}=a+\sum_{r=5}^{9} \epsilon_{r},
$$

and it is the one corresponding to the scalar $\tilde{A}_{4 \mu \nu}$. The $H_{\tilde{\alpha}}$ grading of any field or flux/torsion is given by the scalar product of $\tilde{\alpha}$ by the corresponding weight. It is normalized +1 for the highest weight component of a doublet, +2 for the highest weight component of a triplet and 0 for a singlet. One can easily check that:

$$
\begin{aligned}
\tilde{F}_{\mu \nu \rho \sigma}: & \tilde{\alpha} \cdot W=+2, \\
F_{4 u v w}, H_{4 u v}: & \tilde{\alpha} \cdot W=+1, \\
T_{4 u}^{v}, F_{4 u}: & \tilde{\alpha} \cdot W=0 .
\end{aligned}
$$

This proves the above statement. 
Fluxes, torsion and $\mathbf{T}$-duality. An important advantage of the above mathematical setting is that we can make a simple characterization of the action of $\mathrm{T}$-duality on either fields or fluxes/torsion [29]. Let us consider for the sake of simplicity the dimensional reduction of a Type II theory on a straight torus. The effect of $\mathrm{T}$-duality along the internal direction $X^{r}$ is to transform the corresponding radius $R_{r}$ and the dilaton $\phi$ as follows $\left(\alpha^{\prime}=1\right)$ [30]:

$$
\begin{aligned}
R_{r} & \rightarrow \frac{1}{R_{r}} \Rightarrow \sigma_{r} \rightarrow \sigma_{r}^{\prime}=-\sigma_{r}, \\
\phi & \rightarrow \phi^{\prime}=\phi-\sigma_{r} \quad r=4, \ldots 9 .
\end{aligned}
$$

As a result the $h(\sigma, \phi)$ vector, defined in (6.17), transforms into a new vector $h^{\prime}=h\left(\sigma^{\prime}, \phi^{\prime}\right)$ and this in turn can be absorbed in a transformation of the various $\mathrm{E}_{7(7)}$ weights which contract $h$ in the reduced Lagrangian: $W \cdot h\left(\sigma^{\prime}, \phi^{\prime}\right)=W^{\prime} \cdot h(\sigma, \phi)$. Therefore the effect of T-duality along a number of directions $X^{r}$ is to change the sign of $\epsilon_{r}$ inside the expression of $\mathrm{E}_{7(7)}$ weights:

$$
\text { T-duality along } X^{r} \Rightarrow \epsilon_{r} \rightarrow-\epsilon_{r} \text {. }
$$

This action is consistent with the characterization of T-duality as an automorphism of the group $\mathrm{SO}(6,6)$ which acts on the $\gamma_{s}^{r}, B_{r s}$ moduli $[31,29]$. In particular a T-duality transformation along an odd number of internal directions, which maps Type IIA and Type IIB theories into each other, corresponds to an outer automorphism of the algebra $\mathfrak{s o}(6,6)$.

Given the description of fields, fluxes and torsion in terms of $E_{7(7)}$ weights as in eqs. (6.11), (6.12), (6.20), we can now establish (at a linearized level) the action on them of T-duality. From this framework it follows naturally (see (6.20)) that the flux $H_{r s t}$ and the torsion $T_{r s}^{t}$ are mapped into each other by a $\mathrm{T}$-duality along the direction $X^{t}$ :

$$
\text { T-duality along } X^{t}: \quad H_{r s t} \longrightarrow T_{r s}^{t} \text {. }
$$

This motivates, at the level of maximal supergravity or of truncations of it ${ }^{9}$, the duality correspondence between a vacuum with $\mathrm{H}$-flux in Type II B(A) and a background with internal torsion in Type II A(B).

It should be stressed that the above treatment does not immediately give an interpretation in terms of flux or torsion of all the weights of $\mathbf{9 1 2}$.

\section{Conclusions}

In this paper we have studied the interpretation of the coupling parameters entering the four-dimensional $N=8 \mathrm{~S}-\mathrm{S}$ supergravity in terms of internal background torsion and formfluxes in a dimensional reduction from ten or eleven dimensions. Some of these parameters have an immediate interpretation from a higher dimensional point of view, for some of the others this interpretation is more subtle as they correspond to non-perturbative symmetries of the microscopic theory.

\footnotetext{
${ }^{9}$ For example in the toroidal orientifold models studied in $[11,13]$.
} 
There are various directions for future investigations, which include a similar analysis of theories corresponding to compactifications on more general manifolds with reduced holonomy, the construction of a $D=4$ gauged supergravity deriving from a general torsion background and the effects of a dynamical torsion on this setting. Finally it would be interesting to use our analysis to develop a solution generating technique, in the spirit of [32], which uses the full $\mathrm{E}_{7(7)}$ duality group to connect different supergravity solutions.

\section{A. Some useful relations and definitions about the torsion}

We consider a Riemannian manifold with metric $g_{M N}$. We want to consider an affine connection $\Gamma$ on this manifold, and we require the compatibility of the connection with the metric,

$$
\nabla_{P} g_{M N}=0
$$

If we assume the torsionless condition $\Gamma_{M N}^{P}=\Gamma_{N M}^{P}$, this equation determines, as unique solution, the Levi-Civita connection, given by

$$
\left(\nabla_{M} X\right)^{P}=\partial_{M} X^{P}+\Gamma_{M R}^{P} X^{R}, \quad \Gamma_{M N}^{P}=\frac{1}{2} g^{P R}\left(g_{R M, N}+g_{R N, M}-g_{M N, R}\right),
$$

for the covariant derivative of a vector field $X$.

The torsionlessness condition can be relaxed so the connection coefficients $\Gamma$ for $\nabla$ are not uniquely determined in general. Nevertheless, if we insist on the request of a metric connection, equation (A.1) determines the connection coefficients in terms of the torsion tensor

$$
T_{M N}^{P}=\tilde{\Gamma}_{[M N]}^{P}=\frac{1}{2}\left(\tilde{\Gamma}_{M N}^{P}-\tilde{\Gamma}_{N M}^{P}\right),
$$

as

$$
\tilde{\Gamma}_{M N}^{P}=\Gamma_{M N}^{P}+\frac{1}{2}\left(T_{M N}^{P}-T_{M N}^{P}-T_{N M}^{P}\right),
$$

where $\Gamma_{M N}^{P}$ are the coefficients of the Levi-Civita connection. The indices are raised and lowered with the metric. We notice that the two last terms in (A.3) give a contribution to the symmetric part of the connection. We will denote

$$
K_{M N}^{P}=\frac{1}{2}\left(T_{M N}^{P}-T_{M}^{P}{ }_{N}-T_{N}{ }^{P}{ }_{M}\right) .
$$

Note that $K^{P}{ }_{M N}$ has not definite symmetry in its last two indices, but it is instead antisymmetric in its first two:

$$
K_{M N}^{P}=-K_{M}^{P}{ }_{N}
$$

We will use the formulation of orthonormal frames. The affine connection, defined by $\tilde{\Gamma}_{M N}^{P}$, transforms from the curved frame to the orthonormal one as

$$
\Omega^{B}{ }_{A \mid M}=\tilde{\Gamma}^{R}{ }_{M N} \mathcal{V}_{A}^{N} \mathcal{V}_{R}^{B}+\mathcal{V}_{N}^{B} \partial_{M} \mathcal{V}_{A}^{N}
$$


where $\Omega_{A}^{B}=\Omega^{B}{ }_{A \mid M} d X^{M}$ is the spin connection. If $\tilde{\Gamma}$ is a metric connection, then the spin connection satisfies

$$
\Omega_{A B}=-\Omega_{B A},
$$

where the flat indices are raised and lowered by the flat metric $\eta_{A B}$. The torsion two form is the covariant differential of the identity $\mathbb{1}=\partial_{M} \otimes d x^{M}$, so

$$
T^{M}=d_{\tilde{\nabla}}\left(d X^{M}\right) \equiv d d X^{M}+\tilde{\Gamma}_{N}^{M} \wedge d X^{N}=\tilde{\Gamma}_{R N}^{M} d X^{R} \wedge d X^{N} .
$$

In the orthonormal frame we have $\partial_{M}=\mathcal{V}_{M}^{A} e_{A}$, where $e_{A}$ are the orthonormal vectors. In this basis the torsion tensor is

$$
T^{A}=d \mathcal{V}^{A}+\Omega_{B}^{A} \wedge \mathcal{V}^{B}
$$

If $T^{A}=0$ the spin connection is related through (A.4) to the Levi-Civita connection (A.2). It can be written in terms of the vielbein as

$$
\omega_{M}^{A B}=\mathcal{V}_{C}^{N} \mathcal{V}_{D}^{R} \eta^{A D} \eta^{B C}\left(q_{R \mid M N}-q_{M \mid N R}+q_{N \mid R M}\right)
$$

with

$$
q_{R \mid M N} \equiv \eta_{A B} \mathcal{V}_{R}^{A} \partial_{[M} \mathcal{V}_{N]}^{B}
$$

(for a derivation, see, e.g. [33]). We denote the difference between $\Omega$ and $\omega$ as $\Delta \Omega$. Since the difference between two connections is a tensor we have

$$
\Delta \Omega_{B \mid M}^{A}=\Omega_{B \mid M}^{A}-\omega_{M}^{A B}=\mathcal{V}_{B}^{N} K^{P}{ }_{N M} \mathcal{V}_{P}^{A}=K_{B \mid M}^{A},
$$

and it is related to the torsion form as

$$
T^{A}=d \mathcal{V}^{A}+\Omega_{B}^{A} \wedge \mathcal{V}^{B}=\Delta \Omega_{B \mid M}^{A} \mathcal{V}_{N}^{B} d X^{M} \wedge d X^{N}=K_{[N M]}^{A} d X^{M} \wedge d X^{N}
$$

\section{A.1 Decomposition of $\omega_{B}^{A}$ in terms of $D$-dimensional fields}

Equation (A.5) can be written in the following equivalent form:

$$
\begin{aligned}
\omega_{A B, C} & =q_{A \mid C B}+q_{C \mid A B}+q_{B \mid A C} \\
q_{C \mid A B} & =\mathcal{V}_{[A}{ }^{M} \mathcal{V}_{B]}{ }^{N} \partial_{M} \mathcal{V}_{N C} \\
& =q_{P \mid M N} \mathcal{V}_{C}^{P} \mathcal{V}_{[A}^{M} \mathcal{V}_{B]}^{N}
\end{aligned}
$$

In our case, given (3.2), with all the corresponding fields only depending on $x^{\mu}$, we have

$$
\begin{aligned}
q_{R \mid \underline{m n}} & =0 \\
q_{\underline{n} \mid \mu \underline{m}} & =\frac{1}{2} \eta_{\underline{i j}} \mathcal{V}_{\underline{\underline{\underline{i}}}} \partial_{\mu} \mathcal{V}_{\underline{\underline{m}}}^{\underline{j}}, \\
q_{\nu \mid \mu \underline{m}} & =\frac{1}{2} \eta_{\underline{i j}} \mathcal{V}_{\nu} \underline{\underline{i}} \partial_{\mu} \mathcal{V}_{\underline{\underline{m}}}^{\underline{j}} \\
q_{\underline{\underline{m}} \mid \mu \nu} & =\eta_{\underline{i} \underline{j}} \mathcal{V}_{\underline{\underline{m}}} \partial_{[\mu} \mathcal{V}_{\nu]}^{\underline{j}}, \\
q_{\rho \mid \mu \nu} & =\eta_{\underline{i} \underline{j}} \mathcal{V}_{\rho}^{\underline{i}} \partial_{[\mu} \mathcal{V}_{\nu]}^{\underline{j}}+\eta_{a b} \mathcal{V}_{\rho}^{a} \partial_{[\mu} \mathcal{V}_{\nu]}^{b} .
\end{aligned}
$$




\section{A.2 D-dimensional Kaluza-Klein vectors}

Consider a diffeomorphism on the $x \underline{\underline{m}}$ coordinates which is local with respect to the Ddimensional space-time: $x^{D} \rightarrow x^{\underline{m}}+\xi \underline{\underline{m}}\left(x^{\mu}\right)$. Under such transformation, with the KaluzaKlein assumption, we have:

$$
\begin{aligned}
& \delta \mathcal{V}_{\mu}{ }^{A}=\partial_{\mu} \xi^{\underline{m}} \mathcal{V}_{\underline{m}}{ }^{A} \\
& \delta \mathcal{V}_{\underline{m}}{ }^{A}=0
\end{aligned}
$$

which gives

$$
\delta B \frac{m}{\mu}=\partial_{\mu} \xi^{\underline{m}},
$$

The diffeomorphisms in the $y^{\underline{m}}$ directions appear therefore as gauge transformations for

the abelian vector $B \frac{m}{\mu}$, and, in absence of torsion, the $B^{\underline{m}}$ behave as good abelian vectors in $D$ dimensions.

\section{Acknowledgements}

We are thankful to R. D'Auria and S. Ferrara for reading a preliminary draft of our paper and to G. Bonelli and J. F. Morales for enlightening discussions.

This work has been supported in part by the European Community's Program contract MRTN-CT-2004-005104.

The work of M. A. Ll. has been supported in part by the research grants BFM 200203681 from the Ministerio de Ciencia y Tecnología (Spain) and from EU FEDER funds, and by the research grant GV04B-226 rom the Generalitat Valenciana.

L.A. would like to thank the Physics department of the Politecnico di Torino, the PHTH-division of CERN and the Departamento de Física Teórica, Universidad de Valencia, for their kind hospitality during the preparation of the manuscript.

M. A. Ll. would like to thank the PH-TH-division of CERN for its kind hospitality during the preparation of this work.

\section{References}

[1] J. Scherk and J. H. Schwarz, "How To Get Masses From Extra Dimensions," Nucl. Phys. B 153 (1979) 61.

[2] E.Cremmer, J.Scherk and J.H.Schwarz, "Spontaneously Broken N=8 Supergravity", Phys. Lett. B 84 (1979) 83.

[3] For an exausitive review on flux compactifications see A. R. Frey, "Warped strings: Self-dual flux and contemporary compactifications," arXiv:hep-th/0308156; S. B. Giddings, S. Kachru and J. Polchinski, "Hierarchies from fluxes in string compactifications," Phys. Rev. D 66, 106006 (2002); A. R. Frey and J. Polchinski, Phys. Rev. D65, 126009 (2002); S. Kachru, M. Schulz and S. Trivedi, JHEP 0310, 007 (2003); P. K. Tripathy and S. P. Trivedi, JHEP 0303, 028 (2003); R. Blumenhagen, D. Lust and T. R. Taylor, "Moduli stabilization in chiral type IIB orientifold models with fluxes," Nucl. Phys. B 663, 319 (2003) [arXiv:hep-th/0303016]; M. Berg, M. Haack and B. Kors, Nucl. Phys. B669, 3 (2003); T. R. Taylor and C. Vafa, Phys. Lett. B474, 130 (2000); A. Giryavets, S. Kachru, P. K. Tripathy 
and S. P. Trivedi, "Flux compactifications on Calabi-Yau threefolds," JHEP 0404, 003 (2004) [arXiv:hep-th/0312104]; M. Grana, T. W. Grimm, H. Jockers and J. Louis, "Soft supersymmetry breaking in Calabi-Yau orientifolds with D-branes and fluxes," Nucl. Phys. B 690, 21 (2004) [arXiv:hep-th/0312232]; T. W. Grimm and J. Louis, "The effective action of N = 1 Calabi-Yau orientifolds," arXiv:hep-th/0403067; D. Lust, S. Reffert and S. Stieberger, "Flux-induced soft supersymmetry breaking in chiral type IIb orientifolds with D3/D7-branes," arXiv:hep-th/0406092; D. Lust, P. Mayr, S. Reffert and S. Stieberger, "F-theory flux, destabilization of orientifolds and soft terms on D7-branes," arXiv:hep-th/0501139.

[4] N. Kaloper and R. C. Myers, "The O(dd) story of massive supergravity," JHEP 9905 (1999) 010 [arXiv:hep-th/9901045].

[5] E. Cremmer, S. Ferrara, C. Kounnas and D. V. Nanopoulos, Phys. Lett. B133, 61 (1983).

[6] J. R. Ellis, A. B. Lahanas, D. V. Nanopoulos and K. Tamvakis, Phys. Lett. B134, 429 (1984); J. R. Ellis, C. Kounnas and D. V. Nanopoulos, Nucl. Phys. B247, 373 (1984); A. B. Lahanas and D. V. Nanopoulos, Phys. Rept. 145, 1 (1987); R. Barbieri, E. Cremmer and S. Ferrara, "Flat And Positive Potentials In N=1 Supergravity," Phys. Lett. B 163, 143 (1985).

[7] L. Andrianopoli, R. D'Auria, S. Ferrara and M. A. Lledo, "Gauging of flat groups in four dimensional supergravity," JHEP 0207 (2002) 010 [arXiv:hep-th/0203206].

[8] L. Andrianopoli, R. D'Auria, S. Ferrara, M. A. Lledo; "Duality and Spontaneously Broken Supergravity in Flat Backgrounds," Nucl. Phys. B640 (2002) 63 [arXiv:hep-th/0204145]; L. Andrianopoli, R. D'Auria, S. Ferrara, M. A. Lledo, "Gauged extended supergravity without cosmological constant: no-scale structure and supersymmetry breaking," Int.J.Mod.Phys.A. [arXiv:hep-th/0212141]

[9] S. Kachru, M. B. Schulz, P. K. Tripathy and S. P. Trivedi, "New supersymmetric string compactifications," JHEP 0303 (2003) 061 [arXiv:hep-th/0211182].

[10] R. D'Auria, S. Ferrara and S. Vaula, "N = 4 gauged supergravity and a IIB orientifold with fluxes," New J. Phys. 4 (2002) 71 [arXiv:hep-th/0206241]; R. D’Auria, S. Ferrara, M. A. Lledo and S. Vaula, "No-scale $\mathrm{N}=4$ supergravity coupled to Yang-Mills: The scalar potential and super Higgs effect," Phys. Lett. B 557 (2003) 278 [arXiv:hep-th/0211027]; L. Andrianopoli, R. D'Auria, S. Ferrara, M. A. Lledo, " $N=2$ Super-Higgs, $N=1$ Poincaré Vacua and Quaternionic Geometry," HEP 0301 (2003) 045 [arXiv:hep-th/0212236] L. Andrianopoli, R. D'Auria, S. Ferrara and M. A. Lledo, "4-D gauged supergravity analysis of type IIB vacua on K3 x T**2/Z(2)," JHEP 0303 (2003) 044 [arXiv:hep-th/0302174]; B. de Wit, H. Samtleben and M. Trigiante, "Maximal supergravity from IIB flux compactifications," Phys. Lett. B 583 (2004) 338 [arXiv:hep-th/0311224]; C. Angelantonj, R. D'Auria, S. Ferrara and M. Trigiante, "K3 x T**2/Z(2) orientifolds with fluxes, open string moduli and critical points," Phys. Lett. B 583 (2004) 331 [arXiv:hep-th/0312019];

[11] C. Angelantonj, S. Ferrara and M. Trigiante, "New D = 4 gauged supergravities from N =4 orientifolds with fluxes," JHEP 0310 (2003) 015 [arXiv:hep-th/0306185].

[12] R. D'Auria, S. Ferrara, F. Gargiulo, M. Trigiante and S. Vaula, "N = 4 supergravity Lagrangian for type IIB on $\mathrm{T}^{* *} 6 / \mathrm{Z}(2)$ in presence of fluxes and D3-branes," JHEP 0306 (2003) 045 [arXiv:hep-th/0303049].

[13] C. Angelantonj, S. Ferrara and M. Trigiante, "Unusual gauged supergravities from type IIA and type IIB orientifolds," Phys. Lett. B 582 (2004) 263 [arXiv:hep-th/0310136]; 
[14] J. P. Derendinger, C. Kounnas, P. M. Petropoulos and F. Zwirner, "Superpotentials in IIA compactifications with general fluxes," arXiv:hep-th/0411276.

[15] G. Dall'Agata and S. Ferrara, "Gauged supergravity algebras from twisted tori compactifications with fluxes," hep-th/0502066.

[16] L. Andrianopoli, S. Ferrara and M. A. Lledo, "No-scale D = 5 supergravity from Scherk-Schwarz reduction of D = 6 theories," JHEP 0406 (2004) 018 [arXiv:hep-th/0406018].

[17] R. Rohm, Nucl. Phys. B 237 (1984) 553; C. Kounnas and M. Porrati, Nucl. Phys. B 310 (1988) 355; S. Ferrara, C. Kounnas, M. Porrati and F. Zwirner, Nucl. Phys. B 318 (1989) 75; C. Kounnas and B. Rostand, Nucl. Phys. B 341 (1990) 641; I. Antoniadis, Phys. Lett. B 246 (1990) 377; E. Kiritsis and C. Kounnas, Nucl. Phys. B 503 (1997) 117 [arXiv:hep-th/9703059]; E. Kiritsis, C. Kounnas, P. M. Petropoulos and J. Rizos, Nucl. Phys. B 540 (1999) 87 [arXiv:hep-th/9807067]; I. Antoniadis, E. Dudas and A. Sagnotti, Nucl. Phys. B 544 (1999) 469 [arXiv:hep-th/9807011] and Phys. Lett. B 464 (1999) 38 [arXiv:hep-th/9908023]; I. Antoniadis, G. D’Appollonio, E. Dudas and A. Sagnotti, Nucl. Phys. B 553 (1999) 133 [arXiv:hep-th/9812118]; I. Antoniadis, J. P. Derendinger and C. Kounnas, Nucl. Phys. B 551 (1999) 41 [arXiv:hep-th/9902032]; C. Angelantonj, I. Antoniadis, G. D’Appollonio, E. Dudas and A. Sagnotti, Nucl. Phys. B 572 (2000) 36 [arXiv:hep-th/9911081].

[18] M. Serone and M. Trapletti, "String vacua with flux from freely-acting orbifolds," JHEP 0401 (2004) 012 [arXiv:hep-th/0310245].

[19] A. Strominger, "Superstrings With Torsion," Nucl. Phys. B 274 (1986) 253.

[20] S. Gurrieri, J. Louis, A. Micu and D. Waldram, "Mirror symmetry in generalized Calabi-Yau compactifications," Nucl. Phys. B 654 (2003) 61 [arXiv:hep-th/0211102].

[21] S. Gurrieri and A. Micu, "Type IIB theory on half-flat manifolds," Class. Quant. Grav. 20 (2003) 2181 [arXiv:hep-th/0212278]; R. D'Auria, S. Ferrara, M. Trigiante and S. Vaula, "Gauging the Heisenberg algebra of special quaternionic manifolds," arXiv:hep-th/0410290; R. D'Auria, S. Ferrara, M. Trigiante and S. Vaula, "Scalar potential for the gauged Heisenberg algebra and a non-polynomial antisymmetric tensor theory," arXiv:hep-th/0412063.

[22] G. L. Cardoso, G. Curio, G. Dall'Agata, D. Lust, P. Manousselis and G. Zoupanos, "Non-Kaehler string backgrounds and their five torsion classes," Nucl. Phys. B 652 (2003) 5 [arXiv:hep-th/0211118];

[23] B. de Wit and H. Nicolai, "N=8 Supergravity With Local SO(8) X SU(8) Invariance," Phys. Lett. B 108 (1982) 285.

[24] B. de Wit, H. Samtleben and M. Trigiante, "On Lagrangians and gaugings of maximal supergravities," Nucl. Phys. B 655 (2003) 93 [arXiv:hep-th/0212239];

[25] S. Hojman, M. Rosenbaum, M. P. Ryan and L. C. Shepley, "Gauge Invariance, Minimal Coupling, And Torsion," Phys. Rev. D 17 (1978) 3141.

[26] L. Andrianopoli, R. D'Auria, S. Ferrara, P. Fre and M. Trigiante, "R-R scalars, U-duality and solvable Lie algebras," Nucl. Phys. B 496 (1997) 617 [arXiv:hep-th/9611014]; L. Andrianopoli, R. D'Auria, S. Ferrara, P. Fre, R. Minasian and M. Trigiante, "Solvable Lie algebras in type IIA, type IIB and M theories," Nucl. Phys. B 493 (1997) 249 [arXiv:hep-th/9612202]; E. Cremmer, B. Julia, H. Lu and C. N. Pope, "Dualisation of dualities. I," Nucl. Phys. B 523 (1998) 73 [arXiv:hep-th/9710119]. 
[27] E. Cremmer and B. Julia, "The N=8 Supergravity Theory. 1. The Lagrangian," Phys. Lett. B 80 (1978) 48.

[28] T. Damour, M. Henneaux and H. Nicolai, "Cosmological billiards," Class. Quant. Grav. 20 (2003) R145 [arXiv:hep-th/0212256].

[29] M. Bertolini and M. Trigiante, "Regular R-R and NS-NS BPS black holes," Int. J. Mod. Phys. A 15 (2000) 5017 [arXiv:hep-th/9910237].

[30] see formulas (8.3.30) and (8.3.31) of J. Polchinski, "String Theory", Vol.1, Cambridge University Press, 1998.

[31] H. Lu, C. N. Pope and K. S. Stelle, "Weyl Group Invariance and p-brane Multiplets," Nucl. Phys. B 476 (1996) 89 [arXiv:hep-th/9602140].

[32] O. Lunin and J. Maldacena, "Deforming field theories with U(1) x U(1) global symmetry and their gravity duals," arXiv:hep-th/0502086.

[33] L. Castellani, "Group geometric methods in supergravity and superstring theories," Int. J. Mod. Phys. A 7 (1992) 1583; L. Castellani, R. D'Auria and P. Fre, "Supergravity and superstrings: A Geometric perspective. Vol. 1: Mathematical foundations," SPIRES entry 